\title{
APPROXIMATE AND MEAN APPROXIMATE CONTROLLABILITY PROPERTIES FOR HILFER TIME-FRACTIONAL DIFFERENTIAL EQUATIONS
}

\author{
ERNEST ARAGONES, VALENTIN KEYANTUO, AND MAHAMADI WARMA
}

\begin{abstract}
We study the approximate and mean approximate controllability properties of fractional partial differential equations associated with the so-called Hilfer type time-fractional derivative and a non-negative selfadjoint operator $A_{B}$ with a compact resolvent on $L^{2}(\Omega)$, where $\Omega \subset \mathbb{R}^{N}(N \geq 1)$ is a bounded open set. More precisely, we show that if $0 \leq \nu \leq 1,0<\mu \leq 1$ and $\Omega \subset \mathbb{R}^{N}$ is a bounded open set, then the system

$$
\mathbb{D}_{t}^{\mu, \nu} u+A_{B} u=\left.f\right|_{\omega} \quad \text { in } \Omega \times(0, T),\left(\mathbb{I}_{t}^{(1-\nu)(1-\mu)} u\right)(\cdot, 0)=u_{0} \text { in } \Omega,
$$

is approximately controllable for any $T>0, u_{0} \in L^{2}(\Omega)$ and any non-empty open set $\omega \subset \Omega$. In addition, if the operator $A_{B}$ has the unique continuation property, then the system is also mean approximately controllable. The operator $A_{B}$ can be the realization in $L^{2}(\Omega)$ of a symmetric, non-negative uniformly elliptic second order operator with Dirichlet or Robin boundary conditions, or the realization in $L^{2}(\Omega)$ of the fractional Laplace operator $(-\Delta)^{s}(0<s<1)$ with the Dirichlet exterior condition, $u=0$ in $\mathbb{R}^{N} \backslash \Omega$, or the nonlocal Robin exterior condition, $\mathcal{N}^{s} u+\beta u=0$ in $\mathbb{R}^{N} \backslash \bar{\Omega}$.
\end{abstract}

\section{INTRODUCTION}

Let $\Omega \subset \mathbb{R}^{N}(N \geq 1)$ be a bounded open set with boundary $\partial \Omega$. The main concern of the present paper is to study the controllability properties of a class of fractional (possible space-time) differential equations involving the so-called Hilfer time-fractional derivative. More precisely, we consider the following initial value problem:

$$
\begin{cases}\mathbb{D}_{t}^{\mu, \nu} u+A_{B} u=\left.f\right|_{\omega} & \text { in } \Omega \times(0, T), \\ \left(\mathbb{I}_{t}^{(1-\nu)(1-\mu)} u\right)(\cdot, 0)=u_{0} & \text { in } \Omega,\end{cases}
$$

where $T>0$ and $0 \leq \nu \leq 1,0<\mu \leq 1$ are real numbers, $\mathbb{D}_{t}^{\mu, \nu} u$ denotes the Hilfer time-fractional derivative of order $(\mu, \nu)$ of the function $u$ formally defined by

$$
\mathbb{D}_{t}^{\mu, \nu} u(t):=\mathbb{I}_{t}^{\nu(1-\mu)} \frac{d}{d t}\left(\mathbb{I}_{t}^{(1-\nu)(1-\mu)} u\right)(t), \quad t>0 .
$$

In (1.2), for a real number $\alpha \geq 0, \mathbb{I}_{t}^{\alpha}$ denotes the Riemann-Liouville fractional integral of order $\alpha$ (see (3.3) below for more details).

In (1.1), the operator $A_{B}$ is a non-negative selfadjoint operator on $L^{2}(\Omega)$ with compact resolvent, $u=$ $u(x, t)$ is the state of the system to be controlled and $f=f(x, t)$ is the control function which is localized in a non-empty open set $\omega \subset \Omega$.

Let $f \in L^{2}(\omega \times(0, T))$ and $u_{0} \in C\left((0, T] ; L^{2}(\Omega)\right)$ a solution of the system (1.1). Then the set of reachable states is given by

$$
\mathcal{R}\left(u_{0}, T\right):=\left\{u(\cdot, T): f \in L^{2}(\omega \times(0, T))\right\} .
$$

1991 Mathematics Subject Classification. 93B05, 26A33, 35R11.

Key words and phrases. Fractional differential equations, Mittag-Leffler function, existence and regularity of solutions, interior approximate controllability.

The work of the authors is partially supported by the Air Force Office of Scientific Research under Award NO: FA9550-18$1-0242$ 
We shall say the system (1.1) is null controllable if $0 \in \mathcal{R}\left(u_{0}, T\right)$; exactly controllable if $\mathcal{R}\left(u_{0}, T\right)=L^{2}(\Omega)$; and approximately controllable if $\mathcal{R}\left(u_{0}, T\right)$ is dense in $L^{2}(\Omega)$. It is easy to see that exactly controllable implies null controllable which in turn implies approximately controllable. But the reserve implications are not true in general.

We will say that our system is mean approximately controllable if the set

$$
\left\{\mathbb{I}_{t}^{(1-\nu)(1-\mu)} u(\cdot, T): f \in L^{2}(\omega \times(0, T))\right\}
$$

is dense in $L^{2}(\Omega)$. This is a totally new notion of controllability and is different from the classical approximate controllability in the case $(1-\nu)(1-\mu) \neq 0$; otherwise the two notions coincide as we shall specify below. It is also easy to see that exactly controllable implies null controllable which also implies mean approximately controllable. But we do not know if there is any implication between mean approximately and approximately controllable, except for the already observed fact that for $(1-\mu)(1-\nu)=0$ the two notions coincide.

In our framework, the operator $A_{B}$ can be a realization in $L^{2}(\Omega)$ of a symmetric and uniformly elliptic second order operator with bounded measurable coefficients subject to the Dirichlet or Robin type boundary conditions. Another example for the operator $A_{B}$ is a realization in $L^{2}(\Omega)$ of the fractional Laplace operator $(-\Delta)^{s}(0<s<1)$ with the Dirichlet exterior condition $u=0$ in $\mathbb{R}^{N} \backslash \Omega$, or the nonlocal Robin exterior condition, $\mathcal{N}^{s} u+\beta u=0$ in $\mathbb{R}^{N} \backslash \bar{\Omega}$, where $\beta \in L^{1}\left(\mathbb{R}^{N} \backslash \Omega\right)$ is a non-negative given function and $\mathcal{N}^{s} u$ denotes the nonlocal normal derivative of the function $u$ (see (2.15) for the precise definition). We emphasize that with a small modification of our proofs the Neumann boundary condition (for second order elliptic operators) or the nonlocal Neumann exterior condition $\mathcal{N}^{s} u=0$ in $\mathbb{R}^{N} \backslash \bar{\Omega}$ (for the fractional Laplace operator) can be also included in our framework.

When $\mu=1$, the system (1.1) is the well-known evolution equation of the first order which has been intensively studied. The heat and the Schrödinger equations are included in this framework. The null or/and the approximate controllability of such a system is well-known and has been investigated by several authors when $A_{B}$ is a realization in $L^{2}(\Omega)$ of a uniformly elliptic second order operator with various boundary conditions (Dirichlet, Neumann and Robin). We refer for instance to the monographs [2, 46, and their references for a complete overview. Instead, if $A_{B}$ is a realization in $L^{2}(\Omega)$ of the fractional Laplace operator $(-\Delta)^{s}(0<s<1)$ with Dirichlet, nonlocal Neumann or nonlocal Robin exterior conditions, little is known regarding the fractional heat equation. In one space dimension $(N=1)$, using some properties of the eigenfunctions, eigenvalues and the associated Ingham conditions, it has been shown that the fractional heat equation with the Dirichlet exterior condition is null controllable in any time $T>0$ if and only if $\frac{1}{2}<s<1$. See e.g. [9, 10] for the interior control and [4, 45] for the exterior control, that is, when the control region $\omega$ is localized in $\mathbb{R}^{N} \backslash \bar{\Omega}$. The case of the nonlocal Neumann and Robin exterior conditions remains open due to the lack of information on the associated eigenvalues and eigenfunctions. In space dimension $N \geq 2$, the best possible controllability result available for the fractional heat and wave equations is the approximate controllability recently proved in [26, 28, 43, 44]. The case of the fractional Schrödinger equation in space dimension $N \geq 1$ has been studied in [8] where the author has shown that the system is null controllable for large enough time $T$ if and only if $\frac{1}{2} \leq s<1$. The main tool used is the fractional version of the Pohozaev identity established in [35. This is the only model that we can deal in the multi-dimensional setting.

If $\nu=1$ and $0<\mu<1$, then $\mathbb{D}_{t}^{\mu}:=\mathbb{D}_{t}^{\mu, 0}$ is the Caputo time fractional derivative of order $\mu$. In this context, when $A_{B}=(-\Delta)^{s}(0<s<1)$ with the Dirichlet exterior condition, the exterior approximate controllability properties have been investigated in [4]. Following the same ideas one can also derive some interior approximate controllability results.

The interior approximate controllability in the case $\mathbb{D}_{t}^{\mu}:=\mathbb{D}_{t}^{\mu, 0}$ has been investigated in 18 where the authors have shown that for a symmetric non-negative uniformly elliptic second order operator with the Dirichlet boundary condition, the corresponding fractional diffusion system is approximately controllable in any $T>0, \omega \subset \Omega$ an arbitrary non-empty open set and any $f \in C_{0}^{\infty}(\omega \times(0, T))$.

Most recently, it has been shown in 29] that for any $\mu>0,(\mu \notin \mathbb{N})$ the Caputo type system of order $\mu$ is not null controllable in any time $T>0$, that is, for example if $0<\mu<1$, then there is no control function 
$f$ such that the solution $u$ of the associated system can rest at some time $T>0$. This also shows that such a system cannot be exactly controllable. We would like to emphasize that the proof of the non-null controllability given in [29] also works for the system (1.1) when $\mu \neq 1$.

In the case $\nu=0$ and $0<\mu<1$, the above system becomes the fractional evolution equation with the Riemann-Liouville time-fractional derivative of order $\mu$. Such equations have been intensively studied. The approximate controllability for equations in this class has been studied in 27. In the case of the RiemannLiouville time-fractional derivatives, the initial condition is nonlocal as in (1.1) when $(1-\nu)(1-\mu) \neq 0$.

Motivated by these results, we propose in this paper to investigate the case of the general fractional evolution equation as stated in (1.1) which includes all the above mentioned works.

We summarize the novelties and the main results obtained in the present paper.

- As we have already mentioned, the system considered in the present paper is very general and it includes almost all possible fractional order PDEs. In addition our framework includes not only the classical second order elliptic operators, but it also allows elliptic operators of fractional order like the fractional Laplace operator or the regional fractional Laplace operator (see e.g. 41, 42 for the definition of the regional fractional Laplacian).

- The first main result (Theorem 2.6) states that if $0 \leq \nu<1,0<\mu<1$ and $\omega \subset \Omega$ is an arbitrary non-empty open set, then the system (1.1) is approximately controllable in any time $T>0$. Given that such a system cannot be null controllable if $\mu \neq 1$ (by [29), the approximate controllability is the best possible result that can be expected in the study of the classical controllability properties of the system (1.1).

- Under the above hypothesis on $\omega$ and $0 \leq \nu \leq 1,0<\mu \leq 1$, our second main result (Theorem 2.7) shows that if in addition the operator $A_{B}$ has the unique continuation property (see (2.5)), then the system (1.1) is also mean approximately controllable in any time $T>0$.

- Finally, we show that the mean approximate controllability of (1.1) is equivalent to the uniquecontinuation principle for solutions of the associated adjoint system (3.23), that is,

$$
\left(v \text { solution of (3.23) },\left.v\right|_{\omega \times(0, T)}=0\right) \Longrightarrow v=0 \text { in } \Omega \times(0, T) .
$$

In addition this seems not to be the case for the approximate controllability, unless $(1-\nu)(1-\mu)=0$, in which case mean approximate and approximate controllabilities are the same notions.

As we can observe in the system (1.1), if $(1-\mu)(1-\nu) \neq 0$, then the initial condition is given in terms of the Riemann-Liouville fractional integral. On the contrary, initial conditions for the Caputo derivatives (that is, when $\nu=1$ and $0<\mu<1$ ) are expressed in terms of initial values of integer order derivatives. This allows for a numerical treatment of initial value problems for differential equations of non integer order independently of the chosen definition of the fractional derivative. For this reason, many authors either resort to Caputo derivatives, or use the Riemann-Liouville derivatives but avoid the problem of initial values of fractional derivatives by treating only the case of zero initial conditions.

The interesting paper [25] has provided a series of examples from the field of viscoelasticity which demonstrate that it is possible to attribute physical meaning to initial conditions expressed in terms of RiemannLiouville fractional derivatives (as in (1.1)), and that it is possible to obtain initial values for such initial conditions by appropriate measurements or observations. The mentioned examples include: The Spring-pot model, which is a linear viscoelastic element whose behavior is intermediate between that of an elastic element and a viscous element; a stress relaxation or a general deformation; and an impulse response. For more details we refer to 25 , and the references therein.

Fractional order operators have recently emerged as a modeling alternative in various branches of science and technology. In fact, in many situations, the fractional models reflect better the behavior of the system both in the deterministic and stochastic contexts. A number of stochastic models for explaining anomalous diffusion have been introduced in the literature; among them we mention the fractional Brownian motion; the continuous time random walk; the Lévy flights; the Schneider grey Brownian motion; and more generally, random walk models based on evolution equations of single and distributed fractional order in space (see e.g. 
[15, 22, 32, 36]). In general, a fractional diffusion operator corresponds to a diverging jump length variance in the random walk. We refer to [13, 17, 19, 20, 25] and the references therein for a complete analysis, the derivation and the applications of fractional order operators.

The rest of the paper is organized as follows. In Section 2 we state the main results of the article and give some examples of operators that apply to our situation. Section 3 contains some intermediate results that are needed in the proofs of our main results. More precisely, we prove the existence and uniqueness of weak solutions to the system (1.1) and its associated adjoint system, and give the representation of solutions in terms of series involving the Mittag-Leffler functions. The proofs of the main results are given in Section 4 .

\section{MAin Results AND EXAMPles}

In this section we state the main results of the paper and give some examples. Let $\Omega \subset \mathbb{R}^{N}(N \geq 1)$ be a bounded open set. First, we introduce our assumption on the operator $A_{B}$.

Assumption 2.1. We assume that $A_{B}$ is a non-negative, selfadjoint operator on $L^{2}(\Omega)$ with domain $D\left(A_{B}\right)$, and that the embedding $D\left(A_{B}\right) \hookrightarrow L^{2}(\Omega)$ is compact, where $D\left(A_{B}\right)$ is endowed with the graph norm.

It follows from Assumption 2.1 that $A_{B}$ is given by a bilinear, symmetric, continuous, elliptic and closed form $\mathcal{E}_{B}$ with domain $D\left(\mathcal{E}_{B}\right):=V_{\frac{1}{2}}$ and $\mathcal{E}_{B}(u, u) \geq 0$ for every $u \in V_{\frac{1}{2}}$. Moreover, we have that $A_{B}$ has a compact resolvent, hence, its eigenvalues form a non-decreasing sequence of real numbers $0 \leq \lambda_{1}<$ $\lambda_{2}<\cdots<\lambda_{n} \cdots$ such that $\lim _{n \rightarrow \infty} \lambda_{n}=\infty$. We assume that the first eigenvalue $\lambda_{1}>0$. We denote by $\left(\varphi_{n}\right)_{n \in \mathbb{N}}$ the orthonormal basis of normalized eigenfunctions associated with the eigenvalues $\left(\lambda_{n}\right)_{n \in \mathbb{N}}$. Then $\varphi_{n} \in D\left(A_{B}\right)$ for every $n \in \mathbb{N}$ and $\left(\varphi_{n}\right)_{n \in \mathbb{N}}$ is total in $V_{\frac{1}{2}}$ and in $L^{2}(\Omega)$.

Throughout the remainder of the article, without any mention we shall denote by $V_{-\frac{1}{2}}$ the dual of $V_{\frac{1}{2}}$ with respect to the pivot space $L^{2}(\Omega)$, so that we have the following continuous embeddings: $V_{\frac{1}{2}} \hookrightarrow L^{2}(\Omega) \hookrightarrow V_{-\frac{1}{2}}$. Moreover, $(\cdot, \cdot)_{L^{2}(\Omega)}$ will designate the scalar product in $L^{2}(\Omega)$ and $\langle\cdot, \cdot\rangle_{V_{-\frac{1}{2}}, V_{\frac{1}{2}}}$ will denote the duality pairing between $V_{-\frac{1}{2}}$ and $V_{\frac{1}{2}}$.

2.1. Main results. We first introduce the notion of weak solutions to the system (1.1).

Definition 2.2. Let $u_{0} \in L^{2}(\Omega)$ and $f$ a given function. A function $u$ is said to be a weak solution of the system (1.1), if for every $T>0$, the following properties hold:

- Regularity and initial condition:

$$
\left\{\begin{array}{l}
u \in C\left((0, T] ; V_{\frac{1}{2}}\right) \\
\mathbb{I}_{t}^{(1-\nu)(1-\mu)} u \in C\left([0, T] ; L^{2}(\Omega)\right), \\
\mathbb{D}_{t}^{\mu, \nu} u \in C\left((0, T] ; V_{-\frac{1}{2}}\right)
\end{array}\right.
$$

and $\mathbb{I}_{t}^{(1-\nu)(1-\mu)} u(\cdot, 0)=u_{0}$.

- Variational identity: For every $\varphi \in V_{\frac{1}{2}}$ and a.e. $t \in(0, T)$, we have

$$
\left\langle\mathbb{D}_{t}^{\mu, \nu} u(\cdot, t), \varphi\right\rangle_{V_{-\frac{1}{2}}, V_{\frac{1}{2}}}+\mathcal{E}_{B}(u(\cdot, t), \varphi)-(f(\cdot, t), \varphi)_{L^{2}(\Omega)}=0 .
$$

Now, we introduce our two notions of approximately controllable.

Definition 2.3. The system (1.1) is said to be approximately controllable in time $T>0$, if for every $u_{0}$, $u_{1} \in L^{2}(\Omega)$ and $\varepsilon>0$, there exists a control function $f \in L^{2}\left((0, T) ; L^{2}(\omega)\right)=L^{2}(\omega \times(0, T))$ such that the weak solution $u$ of (1.1) satisfies

$$
\left\|u(\cdot, T)-u_{1}\right\|_{L^{2}(\Omega)} \leq \varepsilon
$$


Definition 2.4. The system (1.1) will be said to be mean approximately controllable in time $T>0$, If for any $u_{0}, u_{1} \in L^{2}(\Omega)$ and $\varepsilon>0$, there exists a control function $f \in L^{2}(\omega \times(0, T))$ such that the weak solution $u$ of (1.1) satisfies

$$
\left\|\mathbb{I}_{t}^{(1-\nu)(1-\mu)} u(\cdot, T)-u_{1}\right\| \leq \varepsilon .
$$

Remark 2.5. We observe that if $(1-\nu)(1-\mu)=0$, then $\mathbb{I}_{t}^{(1-\nu)(1-\mu)} u(\cdot, T)=u(\cdot, T)$, and hence, approximate and mean approximate controllability coincide.

Our main results are the following theorems.

Theorem 2.6. Let $0 \leq \nu<1,0<\mu<1$ and $\omega \subset \Omega$ an arbitrary non-empty open set. Then, the system (1.1) is approximately controllable at any time $T>0$.

Theorem 2.7. Let $0 \leq \nu \leq 1,0<\mu \leq 1$ and $\omega \subset \Omega$ an arbitrary non-empty open set. Assume that the operator $A_{B}$ has the unique continuation property in the sense that,

$$
\text { if } \lambda>0, \varphi \in D\left(A_{B}\right), A_{B} \varphi=\lambda \varphi \text { and } \varphi=0 \text { in } \omega, \text { then } \varphi=0 \text { in } \Omega \text {. }
$$

Then, the system (1.1) is mean approximately controllable.

2.2. Some examples of operators. We conclude this section by giving some examples of operators that satisfy our assumptions.

Example 2.8 (Second orders elliptic operators). We consider the operator $A$ given formally by

$$
A u(x):=-\sum_{i, j=1}^{N} \frac{\partial}{\partial x_{j}}\left(a_{i, j}(x) \frac{\partial u}{\partial x_{i}}(x)\right)+b(x) u(x), \quad x \in \Omega,
$$

where the real-valued coefficients satisfy the following conditions:

$$
a_{i j}(x)=a_{j i}(x), \quad a_{i j} \in W^{1, \infty}(\Omega), 1 \leq i, j \leq N, b \in L^{\infty}(\Omega), b(x) \geq 0, x \in \Omega,
$$

and there is a constant $\rho>0$ such that the following ellipticity condition holds: For a.e. $x \in \Omega$ and all $\xi \in \mathbb{R}^{N}$ we have

$$
\sum_{i, j=1}^{N} a_{i, j}(x) \xi_{i} \xi_{j} \geq \rho|\xi|^{2}
$$

(a) The Dirichlet boundary condition. Let $\Omega \subset \mathbb{R}^{N}$ be an arbitrary bounded open set. Let $A_{D}$ be the selfadjoint operator on $L^{2}(\Omega)$ associated with the closed, bilinear and symmetric form $\mathcal{E}_{D}$ : $W_{0}^{1,2}(\Omega) \times W_{0}^{1,2}(\Omega) \rightarrow \mathbb{R}$ given by

$$
\mathcal{E}_{D}(u, v):=\sum_{i, j=1}^{N} \int_{\Omega} a_{i j} \frac{\partial u}{\partial x_{i}} \frac{\partial v}{\partial x_{j}} d x+\int_{\Omega} b u v d x, \quad u, v \in W_{0}^{1,2}(\Omega) .
$$

More precisely,

$$
\left\{\begin{array}{l}
D\left(A_{D}\right):=\left\{u \in W_{0}^{1,2}(\Omega): \exists w \in L^{2}(\Omega): \mathcal{E}_{D}(u, v)=(w, v)_{L^{2}(\Omega)} \forall v \in W_{0}^{1,2}(\Omega)\right\}, \\
A_{D} u=w .
\end{array}\right.
$$

Then, $A_{D}$ is the realization of $A$ in $L^{2}(\Omega)$ with the Dirichlet boundary condition $B u:=\left.u\right|_{\partial \Omega}=0$ on $\partial \Omega$, and it satisfies all our assumptions. In particular $A_{D}$ has the unique continuation property in the sense of (2.5). 
(b) The Robin boundary condition. Assume that $\Omega \subset \mathbb{R}^{N}$ is a bounded open set with a Lipschitzcontinuous boundary. Let $\beta \in L^{\infty}(\partial \Omega)$ satisfy $\beta(x) \geq \beta_{0}>0$ a.e. on $\partial \Omega$ for some constant $\beta_{0}$. Let $A_{R}$ be the selfadjoint operator on $L^{2}(\Omega)$ associated with the closed, bilinear and symmetric form $\mathcal{E}_{R}: W^{1,2}(\Omega) \times W^{1,2}(\Omega) \rightarrow \mathbb{R}$ given by:

$$
\mathcal{E}_{R}(u, v):=\sum_{i, j=1}^{N} \int_{\Omega} a_{i j} \frac{\partial u}{\partial x_{i}} \frac{\partial v}{\partial x_{j}} d x+\int_{\Omega} b u v d x+\int_{\partial \Omega} \beta u v d \sigma .
$$

As for the Dirichlet boundary condition, we have that

$$
\left\{\begin{array}{l}
D\left(A_{R}\right):=\left\{u \in W^{1,2}(\Omega): \exists w \in L^{2}(\Omega): \mathcal{E}_{R}(u, v)=(w, v)_{L^{2}(\Omega)} \forall v \in W^{1,2}(\Omega)\right\}, \\
A_{R} u=w .
\end{array}\right.
$$

Then, $A_{R}$ is the realization of $A$ in $L^{2}(\Omega)$ with the Robin boundary condition $B u:=\partial_{\nu_{A}} u+\beta u=0$ on $\partial \Omega$. Here,

$$
\partial_{\nu_{A}} u(x):=\sum_{i, j=1}^{N} a_{i j}(x) \frac{\partial u(x)}{\partial x_{i}} \nu_{j}(x) .
$$

The operator $A_{R}$ satisfies all our assumptions and it also enjoys the unique continuation property. For more details we refer to [5, 6, 12, 40] and the references therein.

Before giving some examples involving the fractional Laplace operator, we need to introduce the fractional order Sobolev spaces needed for a rigorous definition of the fractional Laplace operator. Let $\Omega \subset \mathbb{R}^{N}(N \geq 1)$ be an arbitrary bounded open set. For $0<s<1$ we let

$$
W^{s, 2}(\Omega):=\left\{u \in L^{2}(\Omega): \int_{\Omega} \int_{\Omega} \frac{|u(x)-u(y)|^{2}}{|x-y|^{N+2 s}} d x d y<\infty\right\}
$$

and we endow it with the norm

We let

$$
\|u\|_{W^{s, 2}(\Omega)}:=\left(\int_{\Omega}|u|^{2} d x+\int_{\Omega} \int_{\Omega} \frac{|u(x)-u(y)|^{2}}{|x-y|^{N+2 s}} d x d y\right)^{\frac{1}{2}} .
$$

$$
W_{0}^{s, 2}(\bar{\Omega}):=\left\{u \in W^{s, 2}\left(\mathbb{R}^{N}\right): u=0 \text { in } \mathbb{R}^{n} \backslash \Omega\right\}=\left\{u \in W^{s, 2}\left(\mathbb{R}^{N}\right): \operatorname{supp}(u) \subset \bar{\Omega}\right\}
$$

We set

$$
\widetilde{W}_{0}^{s, 2}(\Omega):=\left\{\left.u\right|_{\Omega}: u \in W_{0}^{s, 2}(\bar{\Omega})\right\} .
$$

Next, let $\beta \in L^{1}\left(\mathbb{R}^{N} \backslash \Omega\right)$ be fixed and define the fractional order Sobolev type space

$$
W_{\beta, \Omega}^{s, 2}:=\left\{u: \mathbb{R}^{N} \rightarrow \mathbb{R} \text { measurable : }\|u\|_{W_{\beta, \Omega}^{s, 2}}<\infty\right\},
$$

where

$$
\|u\|_{W_{\beta, \Omega}^{s, 2}}:=\left(\int_{\Omega}|u|^{2} d x+\int_{\mathbb{R}^{N} \backslash \Omega}|u|^{2}|\beta| d x+\iint_{\mathbb{R}^{2 N} \backslash\left(\mathbb{R}^{N} \backslash \Omega\right)^{2}} \frac{|u(x)-u(y)|^{2}}{|x-y|^{N+2 s}} d x d y\right)^{\frac{1}{2}}
$$

and

$$
\mathbb{R}^{2 N} \backslash\left(\mathbb{R}^{N} \backslash \Omega\right)^{2}=(\Omega \times \Omega) \cup\left(\Omega \times\left(\mathbb{R}^{N} \backslash \Omega\right)\right) \cup\left(\left(\mathbb{R}^{N} \backslash \Omega\right) \times \Omega\right) .
$$

The space $W_{\beta, \Omega}^{s, 2}$ has been introduced in 14 to study the nonlocal Neumann problem for $(-\Delta)^{s}$. If $\beta=0$, then we shall denote $W_{0, \Omega}^{s, 2}=W_{\Omega}^{s, 2}$. Then $W_{\beta, \Omega}^{s, 2} \hookrightarrow W_{\Omega}^{s, 2}$ as this is obvious from the above definitions. It has been shown in [14, Proposition 3.1] that $W_{\beta, \Omega}^{s, 2}$ endowed with the norm (2.7) is a Hilbert space. 
For more information on fractional order Sobolev spaces we refer to [13, 14, 17, 23, 41] and the corresponding references.

To introduce the fractional Laplace operator we set

$$
\mathcal{L}_{s}^{1}\left(\mathbb{R}^{N}\right):=\left\{u: \mathbb{R}^{N} \rightarrow \mathbb{R} \text { measurable }: \int_{\mathbb{R}^{N}} \frac{|u(x)|}{(1+|x|)^{N+2 s}} d x<\infty\right\} .
$$

For $u \in \mathcal{L}_{s}^{1}\left(\mathbb{R}^{N}\right)$ and $\varepsilon>0$ we let

$$
(-\Delta)_{\varepsilon}^{s} u(x):=C_{N, s} \int_{\left\{y \in \mathbb{R}^{N}:|y-x|>\varepsilon\right\}} \frac{u(x)-u(y)}{|x-y|^{N+2 s}} d y, \quad x \in \mathbb{R}^{N},
$$

where the normalization constant $C_{N, s}$ is given by

$$
C_{N, s}:=\frac{s 2^{2 s} \Gamma\left(\frac{2 s+N}{2}\right)}{\pi^{\frac{N}{2}} \Gamma(1-s)},
$$

and $\Gamma$ is the usual Euler Gamma function. The fractional Laplace operator $(-\Delta)^{s}$ is defined for $u \in$ $\mathcal{L}_{s}^{1}\left(\mathbb{R}^{N}\right)$ by the formula:

$$
(-\Delta)^{s} u(x):=C_{N, s} \mathrm{P} . \mathrm{V} \cdot \int_{\mathbb{R}^{N}} \frac{u(x)-u(y)}{|x-y|^{N+2 s}} d y=\lim _{\varepsilon \downarrow 0}(-\Delta)_{\varepsilon}^{s} u(x), \quad x \in \mathbb{R}^{N},
$$

provided that the limit exists for a.e. $x \in \mathbb{R}^{N}$. We have that $\mathcal{L}_{s}^{1}\left(\mathbb{R}^{N}\right)$ is the right space for which $v:=(-\Delta)_{\varepsilon}^{s} u$ exists for every $\varepsilon>0$ and $v$ being also continuous at the continuity points of $u$.

For more information on the fractional Laplace operator we refer to [37, 38, 41, 42] and their references.

Example 2.9 (The fractional Laplacian with Dirichlet exterior condition). Firstly, we consider the Dirichlet problem for $(-\Delta)^{s}$, that is, the elliptic equation

$$
(-\Delta)^{s} u=f \quad \text { in } \Omega, \quad u=0 \text { in } \mathbb{R}^{N} \backslash \Omega .
$$

Let $f \in L^{2}(\Omega)$. A function $u \in W_{0}^{s, 2}(\bar{\Omega})$ is said to be a weak solution of (2.10), if

$$
\mathcal{E}(u, v):=\frac{C_{N, s}}{2} \int_{\mathbb{R}^{N}} \int_{\mathbb{R}^{N}} \frac{(u(x)-u(y))(v(x)-v(y))}{|x-y|^{N+2 s}} d y d x=\int_{\Omega} f v d x,
$$

for every $v \in W_{0}^{s, 2}(\bar{\Omega})$. Using the classical Lax-Milgram lemma, it is straightforward to show the existence and uniqueness of weak solutions to the Dirichlet problem (2.10).

Secondly, for a function $u \in L^{2}(\Omega)$ we define its extension $u_{D}$ as follows:

$$
u_{D}(x):= \begin{cases}u(x) & \text { if } x \in \Omega, \\ 0 & \text { if } x \in \mathbb{R}^{N} \backslash \Omega .\end{cases}
$$

Let

$$
D\left(\mathcal{E}_{D}\right):=\left\{u \in L^{2}(\Omega): u_{D} \in W_{0}^{s, 2}(\bar{\Omega})\right\}=\widetilde{W}_{0}^{s, 2}(\Omega),
$$

and $\mathcal{E}_{D}: D\left(\mathcal{E}_{D}\right) \times D\left(\mathcal{E}_{D}\right) \rightarrow \mathbb{R}$ the form given by

$$
\mathcal{E}_{D}(u, v):=\mathcal{E}\left(u_{D}, v_{D}\right)
$$

where $\mathcal{E}$ is given in (2.11). Then, $\mathcal{E}_{D}$ is a densely defined, symmetric and closed bilinear form in $L^{2}(\Omega)$. The selfadjoint operator $(-\Delta)_{D}^{s}$ on $L^{2}(\Omega)$ associated with $\mathcal{E}_{D}$ is given by

$$
\left\{\begin{array}{l}
D\left((-\Delta)_{D}^{s}\right):=\left\{u \in \widetilde{W}_{0}^{s, 2}(\Omega): \exists f \in L^{2}(\Omega) \text { such that } u_{D}\right. \text { is a weak solution of (2.10) } \\
(-\Delta)_{D}^{s} u:=f .
\end{array}\right.
$$


By [38] (see also [11, 37, 41]) the operator $(-\Delta)_{D}^{s}$ has a compact resolvent and its eigenvalues form a nondecreasing sequence of real numbers $0<\lambda_{1} \leq \lambda_{2} \leq \cdots \leq \lambda_{n} \leq \cdots$ satisfying $\lim _{n \rightarrow \infty} \lambda_{n}=\infty$. All the eigenvalues have finite geometric multiplicity. It also follows from [16, Theorem 1.4] that $(-\Delta)_{D}^{s}$ satisfies the unique continuation property in the sense of (2.5).

Example 2.10 (The fractional Laplacian with nonlocal Robin exterior condition). Let $\Omega \subset \mathbb{R}^{N}$ be a bounded open set with a Lipschitz continuous boundary. For $u \in W_{\Omega}^{s, 2}$ we define the nonlocal normal derivative $\mathcal{N}^{s} u$ of $u$ as follows:

$$
\mathcal{N}^{s} u(x):=C_{N, s} \int_{\Omega} \frac{u(x)-u(y)}{|x-y|^{N+2 s}} d y, \quad x \in \mathbb{R}^{N} \backslash \bar{\Omega},
$$

where $C_{N, s}$ is the constant given in (2.8). Clearly, $\mathcal{N}^{s}$ is a nonlocal operator and is well defined on $W_{\Omega}^{s, 2}$.

Let $f \in L^{2}(\Omega), \beta \in L^{1}\left(\mathbb{R}^{N} \backslash \Omega\right)$ a non-negative function and consider the following Robin problem:

$$
(-\Delta)^{s} u=f \quad \text { in } \Omega, \quad \mathcal{N}^{s} u+\beta u=0 \text { in } \mathbb{R}^{N} \backslash \bar{\Omega} .
$$

By a weak solution to (2.16) we mean a function $u \in W_{\beta, \Omega}^{s, 2}$ such that

$$
\frac{C_{N, s}}{2} \iint_{\mathbb{R}^{2 N} \backslash\left(\mathbb{R}^{N} \backslash \Omega\right)^{2}} \frac{(u(x)-u(y))(v(x)-v(y))}{|x-y|^{N+2 s}} d x d y+\int_{\mathbb{R}^{N} \backslash \Omega} \beta u v d x=\int_{\Omega} f v d x
$$

for every $v \in W_{\beta, \Omega}^{s, 2}$. Here also the existence and uniqueness of weak solutions is easy to prove.

For a function $u \in L^{2}(\Omega)$ we define its extension $u_{R}$ as follows:

$$
u_{R}(x):= \begin{cases}u(x) & \text { if } x \in \Omega, \\ \frac{C_{N, s}}{C_{N, s} \rho(x)+\beta(x)} \int_{\Omega} \frac{u(y)}{|x-y|^{N+2 s}} d y & \text { if } x \in \mathbb{R}^{N} \backslash \bar{\Omega},\end{cases}
$$

where

$$
\rho(x):=\int_{\Omega} \frac{1}{|x-y|^{N+2 s}} d y, \quad x \in \mathbb{R}^{N} \backslash \bar{\Omega} .
$$

Since $\partial \Omega$ is a null set (with respect to the $N$-dimensional Lebesgue measure), we have that $u_{R}$ is well defined for every $u \in L^{2}(\Omega)$. In addition, $u_{R}$ satisfies the following Robin exterior condition (see e.g. [1]):

$$
\mathcal{N}^{s} u_{R}+\beta u_{R}=0 \text { in } \mathbb{R}^{N} \backslash \bar{\Omega} \text {. }
$$

Let

$$
D\left(\mathcal{E}_{R}\right):=\left\{u \in L^{2}(\Omega): u_{R} \in W_{\beta, \Omega}^{s, 2}\right\},
$$

and the bilinear form $\mathcal{E}_{R}: D\left(\mathcal{E}_{R}\right) \times D\left(\mathcal{E}_{R}\right) \rightarrow \mathbb{R}$ be given by

$$
\mathcal{E}_{R}(u, v):=\frac{C_{N, s}}{2} \iint_{\mathbb{R}^{2 N} \backslash\left(\mathbb{R}^{N} \backslash \Omega\right)^{2}} \frac{\left(u_{R}(x)-u_{R}(y)\right)\left(v_{R}(x)-v_{R}(y)\right)}{|x-y|^{N+2 s}} d x d y+\int_{\mathbb{R}^{N} \backslash \Omega} \beta u_{R} v_{R} d x .
$$

Then $\mathcal{E}_{R}$ is a closed, symmetric and densely defined bilinear form on $L^{2}(\Omega)$. The selfadjoint operator $(-\Delta)_{R}^{s}$ on $L^{2}(\Omega)$ associated with $\mathcal{E}_{R}$ is given by

$$
\left\{\begin{array}{l}
D\left((-\Delta)_{R}^{s}\right):=\left\{u \in L^{2}(\Omega): u_{R} \in W_{\beta, \Omega}^{s, 2} \exists f \in L^{2}(\Omega) \text { such that } u_{R}\right. \text { is a weak solution of (2.16) } \\
\text { with right hand side } f\}, \\
(-\Delta)_{R}^{s} u:=f .
\end{array}\right.
$$

Then $(-\Delta)_{R}^{s}$ is the realization in $L^{2}(\Omega)$ of $(-\Delta)^{s}$ with the nonlocal Robin exterior condition (2.17). By [11, the operator $(-\Delta)_{R}^{s}$ has compact resolvent and its eigenvalues form a non-decreasing sequence of real numbers $0<\lambda_{1} \leq \lambda_{2} \leq \cdots \leq \lambda_{n} \leq \cdots$ such that $\lim _{n \rightarrow \infty} \lambda_{n}=\infty$. By [28, the operator $(-\Delta)_{R}^{s}$ enjoys the unique continuation property in the sense of (2.5). 


\section{Preliminary Results}

In this section we fix some notations and give some preliminary results that will be used in the proofs of our main results. In particular we introduce the Hilfer time-fractional derivative which is a generalization of the Caputo and Riemann-Liouville time-fractional derivatives. We shall also prove the well-posedness of the system (1.1) and the associated adjoint system.

3.1. Time-fractional derivatives and the Mittag-Leffler functions. Throughout the following, for a real number $\alpha>0$, we let

$$
g_{\alpha}(t):= \begin{cases}\frac{t^{\alpha-1}}{\Gamma(\alpha)} & \text { if } t>0 \\ 0 & \text { if } t \leq 0 .\end{cases}
$$

The (left) Riemann-Liouville fractional integral of order $\alpha>0$ of a locally integrable function $f:(0, \infty) \rightarrow$ $\mathbb{R}$ is defined by

$$
\mathbb{I}_{t}^{\alpha} f(t):=\frac{1}{\Gamma(\alpha)} \int_{0}^{t}(t-\tau)^{\alpha-1} f(\tau) d \tau=\left(g_{\alpha} * f\right)(t), \quad t>0
$$

We let $\left(\mathbb{I}_{t}^{0} f\right)(t):=f(t)$ for all $t>0$. We also note the important semigroup property, $\mathbb{I}_{t}^{\alpha} \mathbb{I}_{t}^{\beta}=\mathbb{I}_{t}^{\alpha+\beta}$.

We have the following property for power functions.

Lemma 3.1. Let $\alpha \geq 0$ and $\beta>-1$. Then,

$$
\mathbb{I}_{t}^{\alpha}\left(t^{\beta}\right)=\frac{\Gamma(\beta+1)}{\Gamma(\alpha+\beta+1)} t^{\alpha+\beta}, \quad t>0 .
$$

Let $X$ be a Banach space and let $f, g:[0, \infty) \rightarrow X$ be locally integrable. Using Laplace transform, we get that for every $t>0$ and $\alpha \geq 0$,

$$
\left(\left(\mathbb{I}_{t}^{\alpha} f\right) * g\right)(t)=\left(f *\left(\mathbb{I}_{t}^{\alpha} g\right)\right)(t) .
$$

The right Riemann-Liouville fractional integral of order $\alpha>0$ of a locally integrable function $u:(0, T) \rightarrow$ $X$ is defined by

$$
\mathbb{I}_{t, T}^{\alpha} u(t):=\frac{1}{\Gamma(\alpha)} \int_{t}^{T}(\tau-t)^{\alpha-1} u(\tau) d \tau, \quad t \in(0, T) .
$$

Now let $0 \leq \nu \leq 1,0<\mu \leq 1$, and let $u:[0, \infty) \rightarrow X$ be a locally integrable function. The (left) Hilfer time-fractional derivative of order $(\mu, \nu)$ is defined by

$$
\mathbb{D}_{t}^{\mu, \nu} u(t):=\mathbb{I}_{t}^{\nu(1-\mu)} \frac{d}{d t}\left(\mathbb{I}_{t}^{(1-\nu)(1-\mu)} u\right)(t), \quad t>0
$$

We observe that if $\nu=0$, then

$$
\mathbb{D}_{t}^{\mu, 0} u(t)=\frac{d}{d t}\left(g_{1-\mu} * u\right)(t)
$$

which is the Riemann-Liouville time-fractional derivative of order $\mu$, while for $\nu=1$, we have that

$$
\mathbb{D}_{t}^{\mu, 1} u(t)=\left(g_{1-\mu} * u^{\prime}\right)(t)=\frac{d}{d t}\left(g_{1-\mu} *(u-u(0))(t),\right.
$$

which corresponds to the Caputo time-fractional derivative of order $\mu$. We refer to [20, Section 2.1] for the justification of the second equality in (3.4) under appropriate conditions. by

Also of interest is the right Hilfer time-fractional derivative of order $(\mu, \nu)(0 \leq \nu \leq 1,0<\mu \leq 1)$ given

$$
\mathbb{D}_{t, T}^{\mu, \nu} u(t):=-\mathbb{I}_{t, T}^{\nu(1-\mu)} \frac{d}{d t}\left(\mathbb{I}_{t, T}^{(1-\nu)(1-\mu)} u\right)(t)
$$


We observe that, if $\mu=1$ and $u$ is differentiable, then

$$
\mathbb{D}_{t}^{1, \nu} u=\frac{d u}{d t} \text { and } \mathbb{D}_{t, T}^{1, \nu} u=-\frac{d u}{d t} .
$$

The right hand derivatives are introduced above since they are needed for the integration by parts formula. Indeed, we have the following integration by parts formula (see e.g. [1, 3, 39]). Let $0 \leq \nu \leq 1,0<\mu \leq 1$. Then,

$$
\int_{0}^{T} v(t) \mathbb{D}_{t}^{\mu, \nu} u(t) d t=\int_{0}^{T} u(t) \mathbb{D}_{t, T}^{\mu, 1-\nu} v(t) d t+\left[\mathbb{I}_{t, T}^{(1-\nu)(1-\mu)} u(t) I_{t}^{\nu(1-\mu)} v(t)\right]_{t=0}^{t=T},
$$

provided that the left and right-hand side expression makes sense. Special cases related to the Caputo and Riemann-Liouville fractional derivatives are easily obtained from the above formula.

The following formula will be useful (see e.g. [39, Theorem 1]). Let $\beta:=\mu+\nu(1-\mu)$. Then

$$
\mathbb{D}_{t}^{\mu, \nu}\left[t^{\beta-1} E_{\mu, \beta}\left(-\omega t^{\mu}\right)\right]=-\omega t^{\beta-1} E_{\mu, \beta}\left(-\omega t^{\mu}\right),
$$

where $E_{\mu, \beta}$ is the Mittag-Leffler function defined in (3.9) below. The proof of (3.7) is a straightforward application of the Laplace transform.

The Laplace transform of the Hilfer time-fractional derivative of a function $f$ is given by:

$$
\mathcal{L}\left(\mathbb{D}_{t}^{\mu, \nu} f\right)(\lambda):=\lambda^{\mu} \mathcal{L}(f)(\lambda)-\lambda^{-\nu(1-\mu)}\left(\mathbb{I}_{t}^{(1-\nu)(1-\mu)} f\right)\left(0^{+}\right) .
$$

For more information on the Hilfer time-fractional derivative we refer to $\underline{39}$ and the references therein.

The Mittag-Leffler function with two parameters is defined as follows:

$$
E_{\alpha, \beta}(z):=\sum_{n=0}^{\infty} \frac{z^{n}}{\Gamma(\alpha n+\beta)}, \quad \alpha>0, \beta \in \mathbb{C}, \quad z \in \mathbb{C} .
$$

It is well-known that $E_{\alpha, \beta}(z)$ is an entire function. This is so even if we allow the parameter set to include $\operatorname{Re}(\alpha)>0$. The following estimate of the Mittag-Leffler function will be useful. Let $0<\alpha<2, \beta \in \mathbb{R}$ and $\kappa$ be such that $\frac{\alpha \pi}{2}<\kappa<\min \{\pi, \alpha \pi\}$. Then there exists a constant $C=C(\alpha, \beta, \kappa)>0$ such that

$$
\left|E_{\alpha, \beta}(z)\right| \leq \frac{C}{1+|z|}, \quad \kappa \leq|\arg (z)| \leq \pi .
$$

In the literature, frequently the notation $E_{\alpha}=E_{\alpha, 1}$ is used. We further note that $E_{1,1}(z)=e^{z}$. The Laplace transform of the Mittag-Leffler function is given by the relation:

$$
\int_{0}^{\infty} e^{-\lambda t} t^{\alpha k+\beta-1} E_{\alpha, \beta}^{(k)}\left( \pm \gamma t^{\alpha}\right) d t=\frac{k ! \lambda^{\alpha-\beta}}{\left(\lambda^{\alpha} \mp \gamma\right)^{k+1}}, \quad \operatorname{Re}(\lambda)>|\gamma|^{1 / \alpha}
$$

Here, $k \in \mathbb{N} \cup\{0\}$ and $\gamma \in \mathbb{R}$.

For more details on fractional derivatives, integrals and the Mittag-Leffler functions we refer to 1, 17, 21, 30, 31, 33, 34, and the references therein.

3.2. Well-posedness of Hilfer type time-fractional evolution equations. Throughout the rest of the paper, without any mention, we assume that the operator $A_{B}$ satisfies Assumption 2.1] Moreover, $\left(\varphi_{n}\right)_{n \in \mathbb{N}}$ denotes the orthonormal basis of eigenfunctions of $A_{B}$ associated with the eigenvalues $\left(\lambda_{n}\right)_{n \in \mathbb{N}}$.

Let $0 \leq \nu \leq 1,0<\mu \leq 1$ and consider the following fractional order homogeneous evolution equation:

$$
\begin{cases}\mathbb{D}_{t}^{\mu, \nu} u+A_{B} u=0 & \text { in } \Omega \times(0, T), \\ \left(\mathbb{I}_{t}^{(1-\nu)(1-\mu)} u\right)(\cdot, 0)=u_{0} & \text { in } \Omega,\end{cases}
$$

where the initial datum $u_{0} \in L^{2}(\Omega)$ and the fractional integrals and derivatives have been defined in Subsection 3.1.

Here is our notion of solutions. 
Definition 3.2. Let $T>0$. We say that a function $u \in C\left((0, T] ; V_{\frac{1}{2}}\right)$ is a weak solution of (3.12), if $\mathbb{D}_{t}^{\mu, \nu} u \in C\left((0, T) ; V_{-\frac{1}{2}}\right), \mathbb{I}_{t}^{(1-\nu)(1-\mu)} u \in C\left([0, T] ; L^{2}(\Omega)\right), \mathbb{I}_{t}^{(1-\nu)(1-\mu)} u(\cdot, 0)=u_{0}$ a.e. in $\Omega$ and

$$
\left\langle\mathbb{D}_{t}^{\mu, \nu} u(\cdot, t), \varphi\right\rangle_{V_{-\frac{1}{2}}, V_{\frac{1}{2}}}+\mathcal{E}_{B}(u(\cdot, t), \varphi)=0,
$$

for every $\varphi \in V_{\frac{1}{2}}$ and a.e. $t \in(0, T)$.

We notice that if $(1-\mu)(1-\nu)=0$, then $\mathbb{I}_{t}^{(1-\nu)(1-\mu)} u(\cdot, 0)=u(\cdot, 0)=u_{0}$.

Next, we define the following operator.

Definition 3.3. Let $0<\mu \leq 1$. Given $u \in L^{2}(\Omega)$ and $t \geq 0$, we let

$$
S_{\mu}(t) u:=\sum_{n=1}^{\infty}\left(u, \varphi_{n}\right)_{L^{2}(\Omega)} E_{\mu, \mu}\left(-\lambda_{n} t^{\mu}\right) \varphi_{n} .
$$

We have the following result.

Lemma 3.4. Let $S_{\mu}(t)$ be the operator defined in (3.13). Then the following assertions hold.

(a) For any fixed $t \geq 0, S_{\mu}(t)$ is a bounded linear operator from $L^{2}(\Omega)$ into $L^{2}(\Omega)$. More precisely, there is a constant $C_{1}>0$ such that for every $u \in L^{2}(\Omega)$ and $t \geq 0$, we have

$$
\left\|S_{\mu}(t) u\right\|_{L^{2}(\Omega)} \leq C_{1}\|u\|_{L^{2}(\Omega)} .
$$

(b) For every $u \in D\left(A_{B}\right)$, we have that $S_{\mu}(t) u \in D\left(A_{B}\right)$ for all $t \geq 0$.

(c) $S_{\mu}(t) S_{\mu}(\tau)=S_{\mu}(\tau) S_{\mu}(t)$ for all $t, \tau \geq 0$.

(d) There is a constant $C_{2}>0$ such that for every $u \in L^{2}(\Omega)$ and $t>0$, we have

$$
\left\|\frac{d S_{\mu}(t) u}{d t}\right\|_{L^{2}(\Omega)} \leq C_{2} t^{-\mu}\|u\|_{L^{2}(\Omega)} .
$$

(e) There is a constant $C_{3}>0$ such that for every $t>0$ and $u \in L^{2}(\Omega)$, we have

$$
\left\|\mathbb{I}_{t}^{\nu(1-\mu)}\left(t^{\mu-1} S_{\mu}(t) u\right)\right\|_{L^{2}(\Omega)} \leq C_{3} t^{-(1-\nu)(1-\mu)}\|u\|_{L^{2}(\Omega)} .
$$

Proof. (a) This assertion follows directly from the definition of the operator $S_{\mu}(t)$ given in (3.13) and the estimate of the Mittag-Leffler function given in (3.10).

(b) Let $u \in D\left(A_{B}\right)$ and $t \geq 0$. Then using (3.10) again, we get that

$$
\left\|S_{\mu}(t) u\right\|_{D\left(A_{B}\right)}^{2} \leq \sum_{n=1}^{\infty}\left|\lambda_{n}\left(S_{\mu}(t) u, \varphi_{n}\right)_{L^{2}(\Omega)}\right|^{2}=\sum_{n=1}^{\infty}\left|\lambda_{n}\left(u, \varphi_{n}\right)_{L^{2}(\Omega)} E_{\mu, \mu}\left(-\lambda_{n} t^{\alpha}\right)\right|^{2} \leq C\|u\|_{D\left(A_{B}\right)}^{2} .
$$

Thus, $S_{\mu}(t) u \in D\left(A_{B}\right)$.

(c) This part is obtained by a simple calculation and using the fact that $\left(\varphi_{n}\right)_{n \in \mathbb{N}}$ is an orthonormal basis of $L^{2}(\Omega)$.

(d) Let $u \in L^{2}(\Omega)$ and $t>0$. Since the series in (3.13) converges in $L^{2}(\Omega)$ uniformly on compact subsets of $[0, \infty)$ (this can be easily justified), we have that

$$
\frac{d S_{\mu}(t) u}{d t}=\sum_{n=1}^{\infty}\left(u, \varphi_{n}\right)_{L^{2}(\Omega)} \frac{d}{d t}\left(E_{\mu, \mu}\left(-\lambda_{n} t^{\mu}\right)\right) \varphi_{n} .
$$

By [24, Theorem 5.1], the derivative of the Mittag-Leffler function is given by

$$
\frac{d}{d t}\left[E_{\mu, \mu}\left(-\lambda_{n} t^{\mu}\right)\right]=\frac{E_{\mu, \mu-1}\left(-\lambda_{n} t^{\mu}\right)+(1-\mu) E_{\mu, \mu}\left(-\lambda_{n} t^{\mu}\right)}{-\mu \lambda_{n} t^{\mu}} .
$$


Using (3.10) and the fact that $\lambda_{n} \geq \lambda_{1}>0$ for every $n \in \mathbb{N}$, we get that there is a constant $C>0$ such that

$$
\left|\frac{d}{d t}\left[E_{\mu, \mu}\left(-\lambda_{n} t^{\mu}\right)\right]\right|=\left|\frac{E_{\mu, \mu-1}\left(-\lambda_{n} t^{\mu}\right)+(1-\mu) E_{\mu, \mu}\left(-\lambda_{n} t^{\mu}\right)}{-\mu \lambda_{n} t^{\mu}}\right| \leq C t^{-\mu}
$$

Thus, the assertion follows by combining (3.15)-(3.16).

(e) This part follows directly by applying Lemma 3.1 The proof is finished.

We have the following result of existence and uniqueness of weak solutions.

Theorem 3.5. Let $0 \leq \nu \leq 1,0<\mu \leq 1$. Then for every $u_{0} \in L^{2}(\Omega)$, the system (3.12) has a unique weak solution u given by

$$
u(\cdot, t)=\sum_{n=1}^{\infty}\left(u_{0}, \varphi_{n}\right)_{L^{2}(\Omega)} t^{-(1-\nu)(1-\mu)} E_{\mu, \mu+\nu(1-\mu)}\left(-\lambda_{n} t^{\mu}\right) \varphi_{n}, t>0,
$$

or equivalently

$$
u(\cdot, t)=\mathbb{I}_{t}^{\nu(1-\mu)}\left(t^{\mu-1} S_{\mu}(t) u_{0}\right), \quad t>0 .
$$

Proof. We give the main ideas of the proof. By Lemma 3.4(e), we have that

$$
\|u(\cdot, t)\|_{L^{2}(\Omega)}=\left\|\mathbb{I}_{t}^{\nu(1-\mu)}\left(t^{\mu-1} S_{\mu}(t) u_{0}\right)\right\|_{L^{2}(\Omega)} \leq C t^{(1-\nu)(\mu-1)}\left\|u_{0}\right\|_{L^{2}(\Omega)} .
$$

We can also easily prove that the series in (3.17) is convergent in $L^{2}(\Omega)$ uniformly in $t \in[\varepsilon, T]$, for every $0<\varepsilon<T$. Thus, we can conclude that $u \in C\left((0, T] ; L^{2}(\Omega)\right)$. Similarly, we can show that $\mathbb{I}_{t}^{(1-\nu)(1-\mu)} u \in$ $C\left([0, T] ; L^{2}(\Omega)\right)$.

Using (3.8) and the Laplace transform, we get that the initial condition is satisfied.

Next, it follows from (3.10) that

$$
\left\|\mathbb{D}_{t}^{\mu, \nu} u(\cdot, t)\right\|_{L^{2}(\Omega)}=\left\|A_{B} u(\cdot, t)\right\|_{L^{2}(\Omega)} \leq C_{2} t^{\nu(1-\mu)-1}\left\|u_{0}\right\|_{L^{2}(\Omega)} .
$$

Using the fact that the series

$$
\sum_{n=1}^{\infty} \lambda_{n}\left(u_{0}, \varphi_{n}\right)_{L^{2}(\Omega)} t^{-(1-\nu)(1-\mu)} E_{\mu, \mu+\nu(1-\mu)}\left(-\lambda_{n} t^{\mu}\right) \varphi_{n},
$$

converges in $L^{2}(\Omega)$ uniformly in $t \in[\varepsilon, T]$, for every $0<\varepsilon<T$, we can also deduce that $\mathbb{D}_{t}^{\mu, \nu} u \in$ $C\left((0, T] ; L^{2}(\Omega)\right) \subset C\left((0, T] ; V_{-\frac{1}{2}}\right)$. The uniqueness of solutions is easy to verify. The proof is finished.

The following result will be needed (see e.g. 26] for the proof).

Lemma 3.6. Let $0<\alpha<2, T>0$ and $\lambda>0$. Then,

$$
\int_{0}^{T} t^{\alpha-1} E_{\alpha, \alpha}\left(-\lambda t^{\alpha}\right) d t=-\frac{1}{\lambda} \int_{0}^{T} \frac{d}{d t} E_{\alpha, 1}\left(-\lambda t^{\alpha}\right) d t=\frac{1}{\lambda}\left(1-E_{\alpha, \alpha}\left(-\lambda T^{\alpha}\right)\right) .
$$

Next, we show the existence and uniqueness of weak solutions to the system (1.1).

Theorem 3.7. Let $0 \leq \nu \leq 1,0<\mu \leq 1, u_{0} \in L^{2}(\Omega)$ and $f \in L^{2}\left((0, T) ; L^{2}(\omega)\right)$. Then the system (1.1) has a unique weak solution $u$ given by

$$
\begin{aligned}
u(\cdot, t)= & \sum_{n=1}^{\infty}\left(u_{0}, \varphi_{n}\right)_{L^{2}(\Omega)} t^{-(1-\nu)(1-\mu)} E_{\mu, \mu+\nu(1-\mu)}\left(-\lambda_{n} t^{\mu}\right) \varphi_{n} \\
& +\sum_{n=1}^{\infty}\left(\int_{0}^{t}\left(f(\cdot, \tau), \varphi_{n}\right)_{L^{2}(\Omega)}(t-\tau)^{\mu-1} E_{\mu, \mu}\left(-\lambda_{n}(t-\tau)^{\mu}\right) d \tau\right) \varphi_{n}
\end{aligned}
$$


or equivalently

$$
u(\cdot, t)=\mathbb{I}_{t}^{\nu(1-\mu)} t^{\mu-1} S_{\mu}(t) u_{0}+\int_{0}^{t}(t-\tau)^{\mu-1} S_{\mu}(t-\tau) f(\cdot, \tau) d \tau .
$$

Proof. The proof follows as the proof of Theorem 3.5, We omit the details for brevity.

3.3. Well-posedness of the associated adjoint system. In order to investigate the controllability properties of the system (1.1), we need to study the following backward system:

$$
\begin{cases}\mathbb{D}_{t, T}^{\mu,(1-\nu)} v+A_{B} v=0 & \text { in } \Omega \times(0, T), \\ \mathbb{I}_{t, T}^{\nu(1-\mu)} v(\cdot, T)=v_{0} & \text { in } \Omega,\end{cases}
$$

which (by using the integration by parts formula (3.6) ) can be viewed as the adjoint system associated with (1.1).

We adopt the following definition of weak solutions to the backward system (3.23).

Definition 3.8. Let $v_{0} \in L^{2}(\Omega)$ and $T>0$. A function $v$ is said to be a weak solution of the system (3.23), if the following properties hold.

- Regularity and final condition:

$$
\left\{\begin{array}{l}
v \in C\left([0, T) ; V_{\frac{1}{2}}\right), \\
\mathbb{I}_{t, T}^{\nu(1-\mu)} v \in C\left([0, T] ; L^{2}(\Omega)\right), \\
\mathbb{D}_{t, T}^{\mu,(1-\nu)} v \in C\left([0, T) ; V_{-\frac{1}{2}}\right),
\end{array}\right.
$$

and $\mathbb{I}_{t, T}^{\nu(1-\mu)} v(\cdot, T)=v_{0}$.

- Variational identity: For every $\varphi \in V_{\frac{1}{2}}$ and a.e. $t \in(0, T)$, we have

$$
\left\langle\mathbb{D}_{t, T}^{\mu,(1-\nu)} v(\cdot, t), \varphi\right\rangle_{V_{-\frac{1}{2}}, V_{\frac{1}{2}}}+\mathcal{E}_{B}(v(\cdot, t), \varphi)=0 .
$$

Next, we show the existence and uniqueness of solutions to the backward system (3.23).

Theorem 3.9. Let $0 \leq \nu \leq 1,0<\mu \leq 1$, and $v_{0} \in L^{2}(\Omega)$. Then the system (3.23) has a unique weak solution $v$ given by

$$
v(\cdot, t)=\sum_{n=1}^{\infty}\left(v_{0}, \varphi_{n}\right)_{L^{2}(\Omega)}(T-t)^{-\nu(1-\mu)} E_{\mu, 1-\nu(1-\mu)}\left(-\lambda_{n}(T-t)^{\mu}\right) \varphi_{n} .
$$

Moreover, the unique weak solution $v$ can be analytically extended to the half-plane

$$
\Sigma_{T}:=\{z \in \mathbb{C}: \operatorname{Re}(z)<T\}
$$

Proof. Since the representation (3.26) and the analytic continuation of solutions are needed in the proof of the main results, we provide more details.

Let $0 \leq \nu \leq 1,0<\mu \leq 1$ and $v_{0} \in L^{2}(\Omega)$. First, we show the uniqueness of solutions. Indeed, let $v$ be a solution of (3.23) with $v_{0}=0$. Taking the inner product of (3.23) with $\varphi_{n}$ and setting $v_{n}(t):=\left(v(t), \varphi_{n}\right)_{L^{2}(\Omega)}$, we get that (given that $A_{B}$ is a selfadjoint operator)

$$
\mathbb{D}_{t, T}^{\mu, 1-\nu} v_{n}(t)=-\lambda_{n} v_{n}(t), \quad \text { for a.e. } t \in(0, T)
$$

Since $\mathbb{I}_{t, T}^{\nu(1-\mu)} v \in C\left([0, T] ; L^{2}(\Omega)\right)$, it follows that $\mathbb{I}_{t, T}^{\nu(1-\mu)} v_{n}(t)=\left(\mathbb{I}_{t, T}^{\nu(1-\mu)} v(\cdot, t), \varphi_{n}\right)_{L^{2}(\Omega)} \in C[0, T]$ and

$$
\left|\mathbb{I}_{t, T}^{\nu(1-\mu)} v_{n}(t)\right|^{2} \leq \sum_{n=1}^{\infty}\left|\mathbb{I}_{t, T}^{\nu(1-\mu)} v_{n}(t)\right|^{2} \leq\left\|\mathbb{I}_{t, T}^{\nu(1-\mu)} v(\cdot, t)\right\|_{L^{2}(\Omega)}^{2} \rightarrow 0 \text { as } t \rightarrow T
$$

This implies that

$$
\mathbb{I}_{t, T}^{\nu(1-\mu)} v_{n}(T)=0
$$


Since the fractional ordinary differential equation (3.27) with the final condition (3.28) has a unique solution $v_{n}$ given by

$$
v_{n}(t)=(T-t)^{-\nu(1-\mu)} E_{\mu, 1-\nu(1-\mu)}\left(-\lambda_{n}(T-t)^{\mu}\right) \mathbb{I}_{t, T}^{\nu(1-\mu)} v_{n}(T),
$$

it follows that $v_{n}(t)=0$ for every $n \in \mathbb{N}$. Since $\left(\varphi_{n}\right)_{n \in \mathbb{N}}$ is a complete orthonormal system in $L^{2}(\Omega)$, we have that $v=0$ in $\Omega \times(0, T)$ and the proof of the uniqueness is complete.

Next, we show the existence of solutions. Let $v_{0, n}:=\left(v_{0}, \varphi_{n}\right)_{L^{2}(\Omega)}, 1 \leq n \leq k$ where $k \in \mathbb{N}$, and set

$$
v_{k}(x, t):=\sum_{n=1}^{k} v_{0, n}(T-t)^{-\nu(1-\mu)} E_{\mu, 1-\nu(1-\mu)}\left(-\lambda_{n}(T-t)^{\mu}\right) \varphi_{n}(x) .
$$

(a) Let $v$ be given by (3.26). We claim that $\mathbb{I}_{t, T}^{\nu(1-\mu)} v \in C\left([0, T] ; L^{2}(\Omega)\right)$. Integrating termwise, we have that (see [39])

$$
\mathbb{I}_{t, T}^{\nu(1-\mu)} v_{k}(\cdot, t)=\sum_{n=1}^{k} v_{0, n} E_{\mu, 1}\left(-\lambda_{n}(T-t)^{\mu}\right) \varphi_{n}
$$

Using (3.10) and the estimates in Lemma 3.6, we have that for every $t \in[0, T]$ and $m, k \in \mathbb{N}$ with $m>k$,

$$
\begin{aligned}
\left\|\mathbb{I}_{t, T}^{\nu(1-\mu)} v_{k}(t)-\mathbb{I}_{t, T}^{\nu(1-\mu)} v_{m}(t)\right\|_{L^{2}(\Omega)}^{2} & =\sum_{n=k+1}^{m}\left|v_{0, n} E_{\mu, 1}\left(-\lambda_{n}(T-t)^{\mu}\right)\right|^{2} \\
& \leq C^{2} \sum_{n=k+1}^{m}\left|v_{0, n}\right|^{2} \rightarrow 0 \text { as } k, m \rightarrow \infty .
\end{aligned}
$$

We have shown that

$$
\sum_{n=1}^{\infty} v_{0, n} E_{\mu, 1}\left(-\lambda_{n}(T-t)^{\mu}\right) \varphi_{n} \rightarrow \mathbb{I}_{t, T}^{\nu(1-\mu)} v(\cdot, t) \text { in } L^{2}(\Omega)
$$

and that the convergence is uniform in $t \in[0, T]$. Hence, $\mathbb{I}_{t, T}^{\nu(1-\mu)} v \in C\left([0, T] ; L^{2}(\Omega)\right)$. Using (3.10) and Lemma 3.6 again, we get that there is a constant $C>0$ such that for every $t \in(0, T)$ we have

$$
\left\|\mathbb{I}_{t, T}^{\nu(1-\mu)} v(\cdot, t)\right\|_{L^{2}(\Omega)}^{2} \leq C^{2}\left\|v_{0}\right\|_{L^{2}(\Omega)}^{2} .
$$

(b) We prove that $\mathbb{D}_{t, T}^{\mu, 1-\nu} v \in C\left([0, T) ; L^{2}(\Omega)\right)$. Since $\mathbb{D}_{t, T}^{\alpha} v(\cdot, t)=-A_{B} v(\cdot, t)$, we have that

$$
\begin{aligned}
\left\|D_{t, T}^{\mu, 1-\nu} v(\cdot, t)\right\|_{L^{2}(\Omega)}^{2} & \leq \sum_{n=1}^{\infty}\left|v_{0, n}\right|^{2}\left|\lambda_{n}(T-t)^{-\nu(1-\mu)} E_{\mu, 1-\nu(1-\mu)}\left(-\lambda_{n}(T-t)^{\mu}\right)\right|^{2} \\
& \leq C(T-t)^{2(\nu \mu-\nu-\mu)}\left\|v_{0}\right\|_{L^{2}(\Omega)}^{2} .
\end{aligned}
$$

Proceeding as above we can deduce that $\mathbb{D}_{t, T}^{\mu, 1-\nu} v \in C\left([0, T) ; L^{2}(\Omega)\right)$.

(c) It follows from (3.29) that

$$
\mathbb{I}_{t, T}^{\nu(1-\mu)} v(\cdot, T)=\sum_{n=1}^{\infty} v_{0, n} \varphi_{n}=v_{0} .
$$

(d) Finally, since $E_{\mu, 1-\nu(1-\mu)}\left(-\lambda_{n} z\right)$ is an entire function, it follows that the function

$$
(T-t)^{-\nu(1-\mu)} E_{\mu, 1-\nu(1-\mu)}\left(-\lambda_{n}(T-t)^{\mu}\right)
$$

can be analytically extended to the half-plane $\Sigma_{T}$. This implies that the function

$$
\sum_{n=1}^{k} v_{0, n}(T-z)^{-\nu(1-\mu)} E_{\mu, 1-\nu(1-\mu)}\left(-\lambda_{n}(T-z)^{\mu}\right) \varphi_{n}
$$


is analytic in $\Sigma_{T}$. Let $\delta>0$ be fixed but otherwise arbitrary. Let $z \in \mathbb{C}$ satisfy $\operatorname{Re}(z) \leq T-\delta$. Then, using Lemma 3.6, we get that

$$
\begin{aligned}
& \left\|\sum_{n=k+1}^{\infty} v_{0, n}(T-z)^{-\nu(1-\mu)} E_{\mu, 1-\nu(1-\mu)}\left(-\lambda_{n}(T-z)^{\mu}\right) \varphi_{n}\right\|_{L^{2}(\Omega)}^{2} \\
\leq & C \sum_{n=k+1}^{\infty}\left|v_{0, n}\right|^{2}|T-z|^{2(\nu \mu-\nu)}\left(\frac{1}{1+\lambda_{n}|T-z|^{\mu}}\right)^{2} \\
\leq & C \delta^{2(\nu \mu-\nu)} \sum_{n=N+1}^{\infty}\left|v_{0, n}\right|^{2} \rightarrow 0 \text { as } k \rightarrow \infty .
\end{aligned}
$$

We have shown that

$$
v(\cdot, z):=\sum_{n=1}^{\infty}\left(v_{0}, \varphi_{n}\right)(T-z)^{\nu \mu-\nu} E_{\mu, 1-\nu(1-\mu)}\left(-\lambda_{n}(T-z)^{\mu}\right) \varphi_{n}
$$

is uniformly convergent in any compact subset of $\Sigma_{T}$. Hence, $v$ is also analytic in $\Sigma_{T}$. The proof of the theorem is finished.

Remark 3.10. We notice that the solution $v$ of the backward system (3.23) satisfies the following additional regularity: There is a constant $C>0$ such that

$$
\|v(\cdot, t)\|_{L^{2}(\Omega)}^{2} \leq C(T-t)^{-2 \nu(1-\mu)}\left\|v_{0}\right\|_{L^{2}(\Omega)}^{2} .
$$

Using (3.32) we can deduce that $v \in C\left([0, T) ; L^{2}(\Omega)\right) \cap L^{1}\left((0, T) ; L^{2}(\Omega)\right)$.

Next, we show that under the assumption that $A_{B}$ has the unique continuation property, the adjoint system (3.23) satisfies the unique continuation principle.

Proposition 3.11. Let $0 \leq \nu \leq 1,0<\mu \leq 1$. Let $v_{0} \in L^{2}(\Omega)$ and let $\omega \subset \Omega$ be an arbitrary non-empty open set. Assume that $A_{B}$ has the unique continuation property in the sense of (2.5). Let $v$ be the unique weak solution of (3.23). If $v=0$ on $\omega \times(0, T)$, then $v=0$ on $\Omega \times(0, T)$.

Proof. Let $v_{0} \in L^{2}(\Omega)$ and let $\omega \subset \Omega$ be an arbitrary non-empty open set. Let $v$ be the unique weak solution of (3.23) and assume that $v=0$ in $\omega \times(0, T)$. Since $v=0$ in $\omega \times(0, T)$ and $v:[0, T) \rightarrow L^{2}(\Omega)$ can be analytically extended to the half-plane $\Sigma_{T}$ (by Theorem[3.9), it follows that for a.e. $x \in \omega$ and $t \in(-\infty, T$ ), we have

$$
v(x, t)=\sum_{n=1}^{\infty}\left(v_{0}, \varphi_{n}\right)_{L^{2}(\Omega)}(T-t)^{-\nu(1-\mu)} E_{\mu, 1-\nu(1-\mu)}\left(-\lambda_{n}(T-t)^{\mu}\right) \varphi_{n}(x)=0 .
$$

Let $\left(\lambda_{k}\right)_{k \in \mathbb{N}}$ be the set of all eigenvalues of the operator $A_{B}$ and let $\left(\psi_{k_{j}}\right)_{1 \leq j \leq m_{k}}$ be an orthonormal basis for $\operatorname{Ker}\left(\lambda_{k}-A_{B}\right)$, where $m_{k}$ is the multiplicity of $\lambda_{k}$. Then (3.33) can be rewritten as

$$
\begin{aligned}
v(x, t) & =\sum_{k=1}^{\infty}\left(\sum_{j=1}^{m_{k}}\left(v_{0}, \psi_{k_{j}}\right)_{L^{2}(\Omega)} \psi_{k_{j}}(x)\right)(T-t)^{-\nu(1-\mu)} E_{\mu, 1-\nu(1-\mu)}\left(-\lambda_{k}(T-t)^{\mu}\right) \\
& =0, \quad x \in \omega, t \in(-\infty, T) .
\end{aligned}
$$


Let $z \in \mathbb{C}$ with $\operatorname{Re}(z):=\eta>0$ and let $M \in \mathbb{N}$. Since the system $\left\{\psi_{k_{j}}\right\}$, for $1 \leq j \leq m_{k}, 1 \leq k \leq M$ is orthonormal, we have that there is a constant $C>0$ such that

$$
\begin{aligned}
& \left\|\sum_{k=1}^{M}\left(\sum_{j=1}^{m_{k}}\left(v_{0}, \psi_{k_{j}}\right)_{L^{2}(\Omega)} \psi_{k_{j}}(x)\right) e^{z(t-T)}(T-t)^{-\nu(1-\mu)} E_{\mu, 1-\nu(1-\mu)}\left(-\lambda_{k}(T-t)^{\mu}\right)\right\|_{L^{2}(\Omega)}^{2} \\
\leq & \sum_{k=1}^{\infty}\left(\sum_{j=1}^{m_{k}}\left|\left(v_{0}, \psi_{k_{j}}\right)_{L^{2}(\Omega)}\right|^{2}\right) e^{2 \eta(t-T)}\left|(T-t)^{-\nu(1-\mu)} E_{\mu, 1-\nu(1-\mu)}\left(-\lambda_{k}(T-t)^{\mu}\right)\right|^{2} \\
\leq & C e^{2 \eta(t-T)}(T-t)^{2 \nu \mu-2 \nu}\left\|v_{0}\right\|_{L^{2}(\Omega)}^{2} .
\end{aligned}
$$

Letting

$$
w_{M}(\cdot, t):=\sum_{k=1}^{M}\left(\sum_{j=1}^{m_{k}}\left(v_{0}, \psi_{k_{j}}\right)_{L^{2}(\Omega)} \psi_{k_{j}}(x)\right) e^{z(t-T)}(T-t)^{-\nu(1-\mu)} E_{\mu, 1-\nu(1-\mu)}\left(-\lambda_{k}(T-t)^{\mu}\right),
$$

we have shown that

$$
\left\|w_{M}(\cdot, t)\right\|_{L^{2}(\Omega)} \leq C e^{\eta(t-T)}(T-t)^{-\nu(1-\mu)}\left\|v_{0}\right\|_{L^{2}(\Omega)} .
$$

The right hand side of (3.35) is integrable over $t \in(-\infty, T)$. More precisely, we have that

$$
\begin{aligned}
\int_{-\infty}^{T} e^{\eta(t-T)}\left[(T-t)^{-\nu(1-\mu)}\left\|v_{0}\right\|_{L^{2}(\Omega)}\right] d t & =\int_{0}^{\infty} e^{-\tau} \frac{\tau^{\mu \nu-\nu+1-1}}{\eta^{1+\nu \mu-\nu}} d \tau \mid v_{0} \|_{L^{2}(\Omega)} \\
& =\frac{\Gamma(1-\nu(1-\mu))}{\eta^{1-\nu(1-\mu)}}\left\|v_{0}\right\|_{L^{2}(\Omega)} .
\end{aligned}
$$

By the Lebesgue dominated convergence theorem, we can deduce that

$$
\begin{aligned}
& \int_{-\infty}^{T} e^{z(t-T)}\left[\sum_{k=1}^{\infty}\left(\sum_{j=1}^{m_{k}}\left(v_{0}, \psi_{k_{j}}\right)_{L^{2}(\Omega)} \psi_{k_{j}}(x)\right)(T-t)^{-\nu(1-\mu)} E_{\mu, 1-\nu(1-\mu)}\left(-\lambda_{k}(T-t)^{\mu}\right)\right] d t \\
= & \sum_{k=1}^{\infty} \sum_{j=1}^{m_{k}} v_{0, k_{j}} \psi_{k_{j}}(x)\left(\int_{-\infty}^{T} e^{z(t-T)}(T-t)^{-\nu(1-\mu)} E_{\mu, 1-\nu(1-\mu)}\left(-\lambda_{k}(T-t)^{\mu}\right) d t\right) \\
= & \sum_{k=1}^{\infty} \sum_{j=1}^{m_{k}}\left(\frac{\left(v_{0}, \psi_{k_{j}}\right)_{L^{2}(\Omega)} z^{-(1-\nu)(1-\mu)}}{z^{\mu}+\lambda_{k}}\right) \psi_{k_{j}}(x), \quad x \in \Omega, \quad \operatorname{Re}(z)>0 .
\end{aligned}
$$

To arrive at (3.36), we have used the fact that

$$
\begin{aligned}
& \int_{-\infty}^{T} e^{z(t-T)}(T-t)^{-\nu(1-\mu)} E_{\mu, \mu-\nu(1-\mu)}\left(-\lambda_{k}(T-t)^{\mu}\right) d t \\
= & \int_{0}^{\infty} e^{-z \tau} \tau^{1-\nu(1-\mu)-1} E_{\mu, 1-\nu(1-\mu)}\left(-\lambda_{k} \tau^{\mu}\right) d \tau=\frac{z^{-(1-\nu)(1-\mu)}}{z^{\mu}+\lambda_{k}}
\end{aligned}
$$

These identities follow from a simple change of variable and (3.11).

It follows from (3.34) and (3.36) that

$$
\sum_{k=1}^{\infty} \sum_{j=1}^{m_{k}}\left(\frac{\left(v_{0}, \psi_{k_{j}}\right)_{L^{2}(\Omega)} z^{-(1-\nu)(1-\mu)}}{z^{\mu}+\lambda_{k}}\right) \psi_{k_{j}}(x)=0, \quad x \in \omega, \operatorname{Re}(z)>0 .
$$


Letting $\eta:=z^{\mu}$, we have shown that

$$
\sum_{k=1}^{\infty} \sum_{j=1}^{m_{k}}\left(\frac{\left(v_{0}, \psi_{k_{j}}\right)_{L^{2}(\Omega)} \eta^{\frac{-(1-\nu)(1-\mu)}{\mu}}}{\eta+\lambda_{k}}\right) \psi_{k_{j}}(x)=0, \quad x \in \omega, \operatorname{Re}(\eta)>0 .
$$

Using the analytic continuation in $\eta$, we have that the identity (3.37) holds for every $\eta \in \mathbb{C} \backslash\left\{-\lambda_{k}\right\}_{k \in \mathbb{N}}$. Taking a suitable small circle about $-\lambda_{l}$ and not passing through nor encircling $\left\{-\lambda_{k}\right\}_{k \neq l}$ and integrating (3.37) on that circle we get that

$$
w_{l}:=\sum_{j=1}^{m_{l}}\left[\left(v_{0}, \psi_{l_{j}}\right)\left(-\lambda_{l}\right)^{-\frac{(1-\nu)(1-\mu)}{\mu}}\right] \psi_{l_{j}}(x)=0, \quad x \in \omega .
$$

Since $\left(A_{B}-\lambda_{l}\right) w_{l}=0$ in $\Omega, w_{l}=0$ in $\omega$, and by assumption $A_{B}$ has the unique continuation property in the sense of (2.5), it follows that $w_{l}=0$ in $\Omega$ for every $l$. Since $\left(\psi_{l_{j}}\right)_{1 \leq j \leq m_{k}}$ is linearly independent in $L^{2}(\Omega)$, we get that $\left(v_{0}, \psi_{l_{j}}\right)_{L^{2}(\Omega)}\left(-\lambda_{l}\right)^{\beta}=0$ for $1 \leq j \leq m_{k}, k \in \mathbb{N}$ and $\beta=-\frac{(1-\nu)(1-\mu)}{\mu}$. This implies that

$$
\begin{aligned}
0 & =\left(-\lambda_{l}\right)^{\beta}\left(v_{0}, \psi_{l_{j}}\right)_{L^{2}(\Omega)} \\
& =\lambda_{l}^{\beta}[\cos (\beta \pi)+i \sin (\beta \pi)]\left(v_{0}, \psi_{l_{j}}\right)_{L^{2}(\Omega)} \\
& =\lambda_{l}^{\beta} \cos (\beta \pi)\left(v_{0}, \psi_{l_{j}}\right)_{L^{2}(\Omega)}+i \lambda_{l}^{\beta} \sin (\beta \pi)\left(v_{0}, \psi_{l_{j}}\right)_{L^{2}(\Omega)} .
\end{aligned}
$$

It follows from (3.38) that $\left(v_{0}, \psi_{l_{j}}\right)_{L^{2}(\Omega)}=0$ for $1 \leq j \leq m_{k}$. Hence, $v=0$ in $\Omega \times(0, T)$. The proof is finished.

\section{Proof of the main Results}

In this section we give the proof of the main results stated in Section 2 , Before proceeding with the proof, we show in the following remark that to study the approximate controllability or the mean approximate controllability of the system (1.1), it suffices to consider the case $u_{0}=0$.

Remark 4.1. Consider the following two systems:

$$
\begin{cases}\mathbb{D}_{t}^{\mu, \nu} v+A_{B} v=\left.f\right|_{\omega \times(0, T)} & \text { in } \Omega \times(0, T), \\ \left(\mathbb{I}_{t}^{(1-\nu)(1-\mu)} v\right)(\cdot, 0)=0 & \text { in } \Omega,\end{cases}
$$

and

$$
\begin{cases}\mathbb{D}_{t}^{\mu, \nu} w+A_{B} w=0 & \text { in } \Omega \times(0, T) \\ \left(\mathbb{I}_{t}^{(1-\nu)(1-\mu)} w\right)(\cdot, 0)=u_{0} & \text { in } \Omega .\end{cases}
$$

Given $u_{0} \in L^{2}(\Omega)$, let $w$ be the weak solution of (4.2). Assume that the system (4.1) is approximately controllable and let $u_{1} \in L^{2}(\Omega)$. Then, for every $\varepsilon>0$, there exists a control function $f \in L^{2}(\omega \times(0, T))$ such that the corresponding unique weak solution $v$ of (4.1) satisfies

$$
\left\|v(\cdot, T)-\left(u_{1}-w(\cdot, T)\right)\right\|_{L^{2}(\Omega)} \leq \varepsilon .
$$

By definition, we have that the function $v+w$ solves the system (1.1), and it follows from (4.3) that

$$
\left\|(v+w)(\cdot, T)-u_{1}\right\|_{L^{2}(\Omega)} \leq \varepsilon .
$$

Hence, (1.1) is approximately controllable. The case of the mean approximate controllability follows similarly.

Proof of Theorem 2.6. Let $0 \leq \nu<1$ and $0<\mu<1$. We recall that the system (1.1) is approximately controllable if $\mathcal{R}\left(u_{0}, T\right)$, with $u_{0}=0$, is dense in $L^{2}(\Omega)$. Since $D\left(A_{B}\right)$ (the domain of $\left.A_{B}\right)$ is dense in $L^{2}(\Omega)$, it is sufficient to show that

$$
D\left(A_{B}\right) \subseteq\left\{u(\cdot, T): f \in L^{2}(\omega \times(0, T))\right\} .
$$


Indeed, assume that $u_{0}=0$ and let $\phi \in D\left(A_{B}\right)$. We set

$$
\psi(\cdot, t):=\frac{\Gamma(\mu)^{2}(T-t)^{1-\mu}}{T}\left[S_{\mu}(T-t)-2 t \frac{d}{d t} S_{\mu}(T-t)\right] \phi .
$$

We claim that $\psi \in L^{2}\left((0, T) ; L^{2}(\Omega)\right)$. Using Lemma 3.4 we get that there is a constant $C>0$ such that for every $t \in(0, T)$ we have

$$
\begin{aligned}
\|\psi(\cdot, t)\|_{L^{2}(\Omega)}^{2} & \leq\left(2 \frac{\Gamma(\mu)^{2}(T-t)^{1-\mu}}{T}\right)^{2}\left[\left\|S_{\mu}(T-t) \phi\right\|_{L^{2}(\Omega)}^{2}+(2 t)^{2}\left\|\frac{d}{d t} S_{\mu}(T-t) \phi\right\|_{L^{2}(\Omega)}^{2}\right] \\
& \leq\left(2 \frac{\Gamma(\mu)^{2}(T-t)^{1-\mu}}{T}\right)^{2}\left(C^{2}+\left(2 t^{1-\mu} C\right)^{2}\right)\|\phi\|_{L^{2}(\Omega)}^{2} .
\end{aligned}
$$

Integrating (4.5) over $(0, T)$ we get that

$$
\begin{aligned}
& \int_{0}^{T}\|\psi(\cdot, t)\|_{L^{2}(\Omega)}^{2} d t \\
\leq & \left(2 \frac{\Gamma(\mu)^{2}\|\phi\|_{L^{2}(\Omega)}}{T}\right)^{2}\left[C^{2} \int_{0}^{T}(T-t)^{2(1-\mu)} d t+(2 C)^{2} \int_{0}^{T} t^{2(1-\mu)}(T-t)^{2(1-\mu)} d t\right] \\
\leq & \left(2 \frac{\Gamma(\mu)^{2}\|\phi\|}{T}\right)^{2}\left[C^{2} \frac{T^{\alpha+1}}{\alpha+1}+(2 C)^{2} T^{2 \alpha+1} \frac{\Gamma(\alpha+1)}{\Gamma(2 \alpha+2)}\right]<\infty,
\end{aligned}
$$

where we have set $\alpha:=2(1-\mu)$, and the claim is proved.

By Theorem 3.7 the system (1.1) with right hand side $\psi$ has a unique weak solution $u$ given by

$$
u(\cdot, t)=\int_{0}^{t}(t-\tau)^{\mu-1} S_{\mu}(t-\tau) \psi(\cdot, \tau) d \tau .
$$

We claim $u(\cdot, T)=\phi$. Using the properties contained in Lemma 3.4 (e), we get that

$$
\begin{aligned}
u(\cdot, T) & =\int_{0}^{T}(T-\tau)^{\mu-1} S_{\mu}(T-\tau) \psi(\cdot, \tau) d \tau \\
& =\frac{\Gamma(\mu)^{2}}{T} \int_{0}^{T}\left(S_{\mu}^{2}(T-\tau) \phi-2 \tau S_{\mu}(T-\tau) \frac{d}{d t} S_{\mu}(T-\tau) \phi\right) d \tau \\
& =\frac{\Gamma(\mu)^{2}}{T}\left(\int_{0}^{T} S_{\mu}^{2}(T-\tau) \phi d \tau-\int_{0}^{T} 2 \tau S_{\mu}(T-\tau) \frac{d}{d t} S_{\mu}(T-\tau) \phi d \tau\right) \\
& =\frac{\Gamma(\mu)^{2}}{T}\left(\int_{0}^{T} S_{\mu}^{2}(T-\tau) \phi d \tau+\int_{0}^{T} \tau \frac{d}{d \tau} S_{\mu}^{2}(T-\tau) \phi d \tau\right) \\
& =\frac{\Gamma(\mu)^{2}}{T}\left(\left[\tau S_{\mu}^{2}(T-\tau) \phi\right]_{\tau=0}^{\tau=T}+\int_{0}^{T} S_{\mu}^{2}(T-\tau) \phi d \tau-\int_{0}^{T} S_{\mu}^{2}(T-\tau) \phi d \tau\right) \\
& =\frac{\Gamma(\mu)^{2}}{T}\left(T S_{\mu}^{2}(0) \phi\right)=\phi,
\end{aligned}
$$

where we also used that $S_{\mu}(0)=\frac{1}{\Gamma(\mu)}$. We have shown (4.4) and the proof is finished.

Proof of Theorem 2.7. $0 \leq \nu \leq 1$ and $0<\mu \leq 1$. Assume that the operator $A_{B}$ has the unique continuation property in the sense of (2.5). Let $u$ be the unique weak solution of (1.1) with $u_{0}=0$ and $v$ the unique weak solution of the adjoint system (3.23) with $v_{0} \in L^{2}(\Omega)$. Then, integrating by parts, we get 
that (by using (3.6) and the fact that $\left.\left(A_{B} u, v\right)_{L^{2}(\Omega)}=\left(u, A_{B} u\right)_{L^{2}(\Omega)}\right)$,

$$
\begin{aligned}
0= & \int_{0}^{T} \int_{\Omega}\left(\mathbb{D}_{t}^{\mu, \nu} u+A_{B} u-f\right) v d x d t \\
= & \int_{0}^{T} \int_{\Omega} v \mathbb{D}_{t}^{\mu, \nu} u d x d t+\int_{0}^{T} \int_{\Omega} v A_{B} u d x d t-\int_{0}^{T} \int_{\omega} f v d x d t \\
= & \int_{0}^{T} \int_{\Omega} u \mathbb{D}_{t, T}^{\mu,(1-\nu)} v d x d t+\int_{\Omega}\left[\mathbb{I}_{t}^{(1-\nu)(1-\mu)} u(x, T) \mathbb{I}_{t, T}^{\nu(1-\mu)} v(x, T)\right] d x \\
& +\int_{0}^{T} \int_{\Omega} u A_{B} v d x d t-\int_{0}^{T} \int_{\omega} f v d x d t \\
= & \int_{0}^{T} \int_{\Omega}\left(\mathbb{D}_{t, T}^{\mu,(1-\nu)} v+A_{B} v\right) u d x d t \\
& +\int_{\Omega}\left[\mathbb{I}_{t}^{(1-\nu)(1-\mu)} u(x, T) \mathbb{I}_{t, T}^{\nu(1-\mu)} v(x, T)\right] d x-\int_{0}^{T} \int_{\omega} f v d x d t \\
= & \int_{\Omega}\left[\mathbb{I}_{t}^{(1-\nu)(1-\mu)} u(x, T) \mathbb{I}_{t, T}^{\nu(1-\mu)} v(x, T)\right] d x-\int_{0}^{T} \int_{\omega} f v d x d t .
\end{aligned}
$$

We have shown that

$$
\int_{\Omega}\left[\mathbb{I}_{t}^{(1-\nu)(1-\mu)} u(x, T) v_{0}\right] d x=\int_{0}^{T} \int_{\omega} f v d x d t
$$

To prove that the set

$$
\left\{\mathbb{I}_{t}^{(1-\nu)(1-\mu)} u(\cdot, T): f \in L^{2}(\omega \times(0, T))\right\}
$$

is dense in $L^{2}(\Omega)$, we have to show that if $v_{0} \in L^{2}(\Omega)$ is such that

$$
\int_{\Omega}\left[\mathbb{I}_{t}^{(1-\nu)(1-\mu)} u(x, T) v_{0}(x)\right] d x=0
$$

for every $f \in L^{2}(\omega \times(0, T))$, then $v_{0}=0$. Indeed, let $v_{0}$ satisfy (4.8). It follows from (4.7) and (4.8) that

$$
\int_{0}^{T} \int_{\omega} f v d x d t=0
$$

for every $f \in L^{2}(\omega \times(0, T))$. By the fundamental lemma of the calculus of variations, we have that

$$
v=0 \text { in } \omega \times(0, T) .
$$

It follows from Proposition 3.11 that

$$
v=0 \text { in } \Omega \times(0, T) .
$$

Since the solution $v$ of (3.23) is unique, it follows that $v_{0}=0$ on $\Omega$. The proof of the theorem is finished.

We conclude the paper with the following remark.

Remark 4.2. We mention the following facts. Let $(1-\nu)(1-\mu) \neq 0$.

(a) Consider the following mapping:

$$
F: L^{2}(\omega \times(0, T)) \rightarrow L^{2}(\Omega), f \mapsto \mathbb{I}_{t}^{(1-\nu)(1-\mu} u(\cdot, T),
$$

where $u$ is the unique weak solution of (1.1) associated to $u_{0}=0$. Then it is easy to see that the system (1.1) is mean approximately controllable in time $T>0$ if and only if the range of $F$, that is, 
$\operatorname{Ran}(F)$ is dense in $L^{2}(\Omega)$. This is equivalent to $\operatorname{Ker}\left(F^{\star}\right)=\{0\}$, where $F^{\star}$ is the adjoint of $F$. It follows from the proof of Theorem 2.7 (more precisely from (4.7)) that $F^{\star}$ is the mapping given by

$$
F^{\star}: L^{2}(\Omega) \rightarrow L^{2}(\omega \times(0, T)),\left.v_{0} \mapsto v\right|_{\omega \times(0, T)},
$$

where $v$ is the unique solution of the adjoint system (3.23). Again $\operatorname{Ker}\left(F^{\star}\right)=\{0\}$ is the unique continuation principle, namely,

$$
\left(v \text { solution of (3.23) },\left.v\right|_{\omega \times(0, T)}=0\right) \Longrightarrow v_{0}=0 \text { in } \Omega .
$$

(b) Now consider the mapping

$$
G: L^{2}(\omega \times(0, T)) \rightarrow L^{2}(\Omega), f \mapsto u(\cdot, T),
$$

where $u$ is the unique weak solution of (1.1) associated with $u_{0}=0$. As above, the system (1.1) is approximately controllable in time $T>0$ if and only if the range of $G$, that is, $\operatorname{Ran}(G)$ is dense in $L^{2}(\Omega)$, and this is equivalent to $\operatorname{Ker}\left(G^{\star}\right)=\{0\}$, where $G^{\star}$ is the adjoint of $G$.

Next, we compute $G^{\star}$. Indeed, let $f \in L^{2}(\omega \times(0, T))$ and $\psi \in L^{2}(\Omega)$. Then,

$$
(G(f), \psi)_{L^{2}(\Omega)}:=\int_{\Omega}(G f)(x, t) \psi(x) d x,
$$

where

$$
(G f)(x, T):=\sum_{n=1}^{\infty}\left(\int_{0}^{T}\left(f(\cdot, t), \varphi_{n}(\cdot)\right)_{L^{2}(\Omega)}(T-t)^{\mu-1} E_{\mu, \mu}\left(-\lambda_{n}(T-t)^{\mu}\right) d t\right) \varphi_{n}(x) .
$$

Now we have that

$$
\begin{aligned}
(G(f), \psi)_{L^{2}(\Omega)} & =\int_{\Omega}\left(\sum_{n=1}^{\infty}\left(\int_{0}^{T}\left(f(\cdot, t), \varphi_{n}(\cdot)\right)_{L^{2}(\Omega)}(T-t)^{\mu-1} E_{\mu, \mu}\left(-\lambda_{n}(T-t)^{\mu}\right) d t\right) \varphi_{n}(x)\right) \psi(x) d x \\
& =\sum_{n=1}^{\infty} \int_{0}^{T}\left(\int_{\Omega}\left(f(\cdot, t), \varphi_{n}(\cdot)\right)_{L^{2}(\Omega)} \varphi_{n}(x) \psi(x) d x\right)(T-t)^{\mu-1} E_{\mu, \mu}\left(-\lambda_{n}(T-t)^{\mu}\right) d t \\
& =\sum_{n=1}^{\infty} \int_{0}^{T}\left(\int_{\Omega} \int_{\Omega} f(y, t) \varphi_{n}(y) \varphi_{n}(x) \psi(x) d y d x\right)(T-t)^{\mu-1} E_{\mu, \mu}\left(-\lambda_{n}(T-t)^{\mu}\right) d t
\end{aligned}
$$

Using (3.21) in Theorem 3.7 and applying Fubini's theorem we get that for all $\psi \in L^{2}(\Omega), 0 \leq t<T$ and for a.e $x \in \Omega$,

$$
\begin{aligned}
(G(f), \psi)_{L^{2}(\Omega)}= & \sum_{n=1}^{\infty} \int_{0}^{T}\left(\int_{\Omega}\left(\varphi_{n}(\cdot), \Psi(\cdot)\right)_{L^{2}(\Omega)} f(y, t) \varphi_{n}(y) d y\right)(T-t)^{\mu-1} E_{\mu, \mu}\left(-\lambda_{n}(T-t)^{\mu}\right) d t \\
& =\int_{\Omega} \int_{0}^{T}\left(\sum_{n=1}^{\infty}\left(\varphi_{n}(\cdot), \psi(\cdot)\right)_{L^{2}(\Omega)}(T-t)^{\mu-1} E_{\mu, \mu}\left(-\lambda_{n}(T-t)^{\mu}\right)\right) \varphi_{n}(y) f(y, t) d t d y \\
& =\left(f,\left(G^{*} \psi\right)\right)_{L^{2}(\Omega)}
\end{aligned}
$$

We have shown that for all $\psi \in L^{2}(\Omega), 0 \leq t<T$ and for a.e $x \in \Omega$,

$$
\left(G^{*} \psi\right)(x, t)=\sum_{n=1}^{\infty}\left(\varphi_{n}, \psi\right)_{L^{2}(\Omega)}(T-t)^{\mu-1} E_{\mu, \mu}\left(-\lambda_{n}(T-t)^{\mu}\right) \varphi(x) .
$$

We can see from (4.9) that $\operatorname{Ker}\left(G^{\star}\right)$ is not related to the adjoint system (3.23). For that reason the approximate controllability of the system (1.1) (in the case $0 \leq \nu<1$ and $0<\mu<1$ ) is not directly related to the unique continuation principle for the adjoint system (3.23). 


\section{REFERENCES}

[1] O. P. Agrawal. Fractional variational calculus in terms of Riesz fractional derivatives. J. Phys. A, 40(24):6287-6303, 2007.

[2] F. Alabau-Boussouira, R. Brockett, O. Glass, J. Le Rousseau, and E. Zuazua. Control of partial differential equations, volume 2048 of Lecture Notes in Mathematics. Springer, Heidelberg; Fondazione C.I.M.E., Florence, 2012. Lectures from the CIME Course held in Cetraro, July 19-23, 2010, Edited by Piermarco Cannarsa and Jean-Michel Coron, Fondazione CIME/CIME Foundation Subseries.

[3] R. Almeida and D. F. M. Torres. Necessary and sufficient conditions for the fractional calculus of variations with Caputo derivatives. Commun. Nonlinear Sci. Numer. Simul., 16(3):1490-1500, 2011.

[4] H. Antil, U. Biccari, R. Ponce, M. Warma, and S. Zamorano. Controllability properties from the exterior under positivity constraints for a 1-d fractional heat equation. arXiv preprint arXiv:1910.14529, 2019.

[5] W. Arendt and M. Warma. Dirichlet and Neumann boundary conditions: What is in between? volume 3, pages 119-135. 2003. Dedicated to Philippe Bénilan.

[6] W. Arendt and M. Warma. The Laplacian with Robin boundary conditions on arbitrary domains. Potential Anal., 19(4):341-363, 2003.

[7] E. Bazhlekova. Fractional evolution equations in Banach spaces. Ph.D. Thesis, Eindhoven University of Technology, 2001.

[8] U. Biccari. Internal control for non-local Schrödinger and wave equations involving the fractional Laplace operator. arXiv preprint arXiv:1411.7800, 2018.

[9] U. Biccari and V. Hernández-Santamaria. Controllability of a one-dimensional fractional heat equation: theoretical and numerical aspects. IMA J. Math. Control Inf., 36(4):1199-1235, 2019.

[10] U. Biccari, M. Warma, and E. Zuazua. Controllability of the one-dimensional fractional heat equation under positivity constraints. Communications on Pure and Applied Analysis, 19(4):1949-1978, 2020.

[11] B. Claus and M. Warma. Realization of the fractional Laplacian with nonlocal exterior conditions via forms method. $J$. Evol. Equ., 2020, DOI: https://doi.org/10.1007/s00028-020-00567-0.

[12] D. Daners. Robin boundary value problems on arbitrary domains. Trans. Amer. Math. Soc., 352(9):4207-4236, 2000.

[13] E. Di Nezza, G. Palatucci, and E. Valdinoci. Hitchhiker's guide to the fractional Sobolev spaces. Bull. Sci. Math., 136(5):521-573, 2012.

[14] S. Dipierro, X. Ros-Oton, and E. Valdinoci. Nonlocal problems with Neumann boundary conditions. Rev. Mat. Iberoam., 33(2):377-416, 2017.

[15] A. A. Dubkov, B. Spagnolo, and V. V. Uchaikin. Lévy flight superdiffusion: an introduction. Internat. J. Bifur. Chaos Appl. Sci. Engrg., 18(9):2649-2672, 2008.

[16] M. M. Fall and V. Felli. Unique continuation property and local asymptotics of solutions to fractional elliptic equations. Comm. Partial Differential Equations, 39(2):354-397, 2014.

[17] A. Fiscella, R. Servadei, and E. Valdinoci. Density properties for fractional Sobolev spaces. Ann. Acad. Sci. Fenn. Math., 40(1):235-253, 2015.

[18] K. Fujishiro and M. Yamamoto. Approximate controllability for fractional diffusion equations by interior control. Appl. Anal., 93(9):1793-1810, 2014.

[19] C. G. Gal and M. Warma. Nonlocal transmission problems with fractional diffusion and boundary conditions on non-smooth interfaces. Comm. Partial Differential Equations, 42(4):579-625, 2017.

[20] C. G. Gal and M. Warma. Fractional in time semilinear parabolic equations and applications, volume 84 of Mathématiques and Applications series. Springer, Berlin, Heidelberg, 2020.

[21] R. Gorenflo and F. Mainardi. Fractional calculus: integral and differential equations of fractional order. In Fractals and fractional calculus in continuum mechanics (Udine, 1996), volume 378 of CISM Courses and Lect., pages 223-276. Springer, Vienna, 1997.

[22] R. Gorenflo, F. Mainardi, and A. Vivoli. Continuous-time random walk and parametric subordination in fractional diffusion. Chaos Solitons Fractals, 34(1):87-103, 2007.

[23] P. Grisvard. Elliptic problems in nonsmooth domains, volume 24 of Monographs and Studies in Mathematics. Pitman (Advanced Publishing Program), Boston, MA, 1985.

[24] H. J. Haubold, A. M. Mathai, and R. K. Saxena. Mittag-Leffler functions and their applications. J. Appl. Math., pages Art. ID 298628, 51, 2011.

[25] N. Heymans and I. Podlubny. Physical interpretation of initial conditions for fractional differential equations with riemannliouville fractional derivatives. Rheologica Acta, 45(5):765-771, 2006.

[26] V. Keyantuo and M. Warma. On the interior approximate controllability for fractional wave equations. Discrete Contin. Dyn. Syst., 36(7):3719-3739, 2016.

[27] Z. Liu and X. Li. Approximate controllability of fractional evolution systems with Riemann-Liouville fractional derivatives. SIAM J. Control Optim., 53(4):1920-1933, 2015.

[28] C. Louis-Rose and M. Warma. Approximate controllability from the exterior of space-time fractional wave equations. Applied Mathematics and Optimization, pages 1-44, 2018, DOI: https://doi.org/10.1007/s00245-018-9530-9.

[29] Q. Lü and E. Zuazua. On the lack of controllability of fractional in time ODE and PDE. Math. Control Signals Systems, 28(2):Art. 10, 21, 2016. 
[30] F. Mainardi. Fractional calculus: some basic problems in continuum and statistical mechanics. In Fractals and fractional calculus in continuum mechanics (Udine, 1996), volume 378 of CISM Courses and Lect., pages 291-348. Springer, Vienna, 1997.

[31] F. Mainardi and R. Gorenflo. On Mittag-Leffler-type functions in fractional evolution processes. J. Comput. Appl. Math., 118(1-2):283-299, 2000. Higher transcendental functions and their applications.

[32] B. B. Mandelbrot and J. W. Van Ness. Fractional Brownian motions, fractional noises and applications. SIAM Rev., 10:422-437, 1968.

[33] K. S. Miller and B. Ross. An introduction to the fractional calculus and fractional differential equations. A WileyInterscience Publication. John Wiley \& Sons, Inc., New York, 1993.

[34] I. Podlubny. Fractional differential equations, volume 198 of Mathematics in Science and Engineering. Academic Press, Inc., San Diego, CA, 1999. An introduction to fractional derivatives, fractional differential equations, to methods of their solution and some of their applications.

[35] X. Ros-Oton and J. Serra. The Pohozaev identity for the fractional Laplacian. Arch. Ration. Mech. Anal., 213(2):587-628, 2014.

[36] W. R. Schneider. Grey noise. In Stochastic processes, physics and geometry (Ascona and Locarno, 1988), pages 676-681. World Sci. Publ., Teaneck, NJ, 1990.

[37] R. Servadei and E. Valdinoci. Variational methods for non-local operators of elliptic type. Discrete Contin. Dyn. Syst., 33(5):2105-2137, 2013.

[38] R. Servadei and E. Valdinoci. On the spectrum of two different fractional operators. Proc. Roy. Soc. Edinburgh Sect. A, 144(4):831-855, 2014.

[39] Z. Tomovski, R. Hilfer, and H. M. Srivastava. Fractional and operational calculus with generalized fractional derivative operators and Mittag-Leffler type functions. Integral Transforms Spec. Funct., 21(11):797-814, 2010.

[40] M. Warma. The Laplacian with general Robin boundary conditions. PhD Dissertation, University of Ulm, 2002.

[41] M. Warma. The fractional relative capacity and the fractional Laplacian with Neumann and Robin boundary conditions on open sets. Potential Anal., 42(2):499-547, 2015.

[42] M. Warma. The fractional Neumann and Robin type boundary conditions for the regional fractional $p$-Laplacian. NoDEA Nonlinear Differential Equations Appl., 23(1):Art. 1, 46, 2016.

[43] M. Warma. On the approximate controllability from the boundary for fractional wave equations. Appl. Anal., 96(13):2291$2315,2017$.

[44] M. Warma. Approximate controllability from the exterior of space-time fractional diffusive equations. SIAM J. Control Optim., 57(3):2037-2063, 2019.

[45] M. Warma and S. Zamorano. Null controllability from the exterior of a one-dimensional nonlocal heat equation. Control and Cybernetics, 2019, to appear.

[46] E. Zuazua. Controllability of partial differential equations. 3ème cycle. Castro Urdiales, Espagne, 2006.

E. Aragones, University of Puerto Rico, Rio Piedras Campus, Faculty of Natural Sciences, Department of Mathematics, 17 University AVE. STE 1701, San Juan PR 00925-2537 (USA)

E-mail address: ernest.aragones@upr.edu

V. Keyantuo, University of Puerto Rico, Rio Piedras Campus, Faculty of Natural Sciences, Department of Mathematics, 17 University AVE. STE 1701, San Juan PR 00925-2537 (USA)

E-mail address: valentin.keyantuo1@upr.edu

M. Warma, Department of Mathematical Sciences, George Mason University. Fairfax, VA 22030 (USA).

E-mail address: mwarma@gmu.edu 